\title{
Positive Lyapunov exponent for random perturbations of predominantly expanding multimodal circle maps
}

\author{
Alex Blumenthal and Yun Yang
}

\begin{abstract}
We study the effects of independent, identically distributed random perturbations of amplitude $\varepsilon>0$ on the asymptotic dynamics of one-parameter families $\left\{f_{a}: S^{1} \rightarrow S^{1}, a \in[0,1]\right\}$ of smooth multimodal maps which are "predominantly expanding", i.e., $\left|f_{a}^{\prime}\right| \gg 1$ away from small neighborhoods of the critical set $\left\{f_{a}^{\prime}=0\right\}$. We obtain, for any $\varepsilon>0$, a checkable, finite-time criterion on the parameter $a$ for random perturbations of the map $f_{a}$ to exhibit (i) a unique stationary measure and (ii) a positive Lyapunov exponent comparable to $\int_{S^{1}} \log \left|f_{a}^{\prime}\right| d x$. This stands in contrast with the situation for the deterministic dynamics of $f_{a}$, the chaotic regimes of which are determined by typically uncheckable, infinite-time conditions. Moreover, our finite-time criterion depends on only $k \sim \log \left(\varepsilon^{-1}\right)$ iterates of the deterministic dynamics of $f_{a}$, which grows quite slowly as $\varepsilon \rightarrow 0$.
\end{abstract}

\section{Introduction and statement of results}

A fundamental goal in dynamical systems is to determine the asymptotic behavior of various dynamical systems. Away from the uniformly expanding, Anosov and Axiom A settings, maps can have "mixed" dynamical behavior, e.g., hyperbolicity on some parts of phase space and contractive behavior on others. On the collection of maps with this "mixed" behavior, various dynamical regimes (e.g., asymptotically stable orbits with large basins of attraction versus more "chaotic" asymptotic behavior) can be intermingled, in the space of maps, in an extremely convoluted way.

These issues are already present in the deceptively simple example of the oneparameter family of quadratic maps $f_{a}:[0,1] \rightarrow[0,1], f_{a}(x):=\operatorname{ax}(1-x)$ for $a \in[0,4]$. Let us agree to say that for a parameter $a \in[0,4]$, the map $f_{a}$ is regular if phase space $[0,1]$ is covered Lebesgue almost-surely by the basins of periodic sinks, while $f_{a}$ is chaotic if it possesses a unique absolutely continuous invariant measure (a.c.i.m.) with a positive Lyapunov exponent. For the family $\left\{f_{a}\right\}$, it is known (e.g., [37] and many others) that the parameter space $[0,4]$ is Lebesgue-almost surely partitioned into two sets, $\mathcal{A} \cup \mathcal{B}$, with the following properties:

- For all $a \in \mathcal{A}$, the map $f_{a}$ is regular, and for all $a \in \mathscr{B}$, the map $f_{a}$ is chaotic.

- The set $\mathcal{A}$ is open and dense in [0,4], while $\mathscr{B}$ has positive Lebesgue measure. In particular, every $a \in \mathscr{B}$ is the limit point of a sequence $\left\{a_{n}\right\} \subset \mathcal{A}$. 
In particular, the chaotic property is extremely structurally unstable with respect to the parameter $a$ : any $a \in \mathscr{B}$ is the limit point of a sequence $\left\{a_{n}\right\} \subset \mathcal{A}$.

Aside from "exceptional" cases (e.g., $a=4$ ), it is typically impossible to rigorously determine, even with the help of a computer, the dynamical regime corresponding to a given parameter $a \in[0,4]$, as this determination would require infinite-precision knowledge of infinite-length trajectories. For the quadratic family and other families of onedimensional maps with mixed expansion and contraction, the core issue is the difficulty in ruling out the formation of sinks of high period: even if, for a given $a$, sinks of period $\leq N$ are ruled out for some extremely large $N$, one cannot rule out the existence of a sink of period $N+1$ or greater. Indeed, the trajectory of a sink of large period may "look" chaotic before the full period has elapsed.

Although fewer results are known for higher-dimensional models, one anticipates a similar degree of convoluted intermingling of dynamical regimes: see, e.g., the class of examples now known as Newhouse phenomena ([41]). A somewhat more complete account of coexistence phenomena is available for the famous Chirikov standard map family ([15]), a one-parameter family $\left\{F_{L}, L>0\right\}$ of volume-preserving maps on the torus $\mathbb{T}^{2}$ exhibiting simultaneously both strong hyperbolicity and elliptic-type behavior on phase space. As the parameter $L$ increases, so too does the proportion of phase space on which $F_{L}$ is hyperbolic, as well as the "strength" of this hyperbolicity. However, even for large $L$, a small amount of elliptic-type behavior is intermingled with hyperbolic behavior in the parameter space. Indeed, for a residual set of large $L$, it is known that elliptic islands for $F_{L}$ are approximately $L^{-1}$-dense in $\mathbb{T}^{2}$ ([17]; see also [18]), while the set of points with a positive Lyapunov exponent has Hausdorff dimension 2 and is approximately $L^{-1 / 3}$-dense in $\mathbb{T}^{2}$ ([24]). To the authors' knowledge, it is still not known whether $F_{L}$ has positive metric entropy (equivalently, a positive Lyapunov exponent on a positive-volume set) for any fixed value of $L$.

A similar situation exists for the Hénon family of diffeomorphisms $f_{a, b}(x, y):=$ $\left(1-a x^{2}+y, b x\right)$ for real parameters $a, b$, introduced by Hénon ([25]) as a toy model capturing the dynamics of Poincaré sections of the Lorenz model ([30]) in certain parameter ranges. Note that the singular limit $b \rightarrow 0$ corresponds with the quadratic map family. Of particular interest are the "classical parameters" $a=1.4, b=0.3$ at which a wealth of numerical evidence suggests $f_{a, b}$ admits a chaotic strange attractor (see, e.g., the original work [25]). This remains a major open problem and is likely to be quite difficult: see, e.g., [21,22] which establish the existence of parameters close to $(a, b)=(1.4,0.3)$ at which the attractor degenerates into periodic sinks. Another known difficulty is the mechanism of unfurling of homoclinic tangencies ([40]); for the Hénon map specifically, see for example [6] and the references therein. At present, the existence of strange attractors for $f_{a, b}$ is only known for perturbatively small values of $b$ ([5,39]). This work has since been substantially generalized to a framework for establishing the existence of rank one strange attractors in the work of Wang, Young and others in a variety of contexts, e.g., near limit cycles subjected to time-periodic forcing with long period ([34, 42, 47, 49]). We emphasize that these constructions are quite challenging, and do not explicitly identify 
parameters at which the strange attractors exist; instead, a parametrized family of maps is considered, and a nonempty set of parameters (a positive-volume Cantor set) is identified at which a strange attractor exists.

Random perturbations. The real world is inherently noisy, and so it is natural to consider independent, identically distributed (IID) random perturbations of otherwise deterministic dynamics and seek to understand the corresponding asymptotic behavior. For concreteness, let us consider a smooth, deterministic map $f: S^{1} \rightarrow S^{1}$ and assume that $\left|f^{\prime}\right|>2$ on all but a small neighborhood of the critical set $\left\{f^{\prime}=0\right\}$ for $f$.

Parametrizing $S^{1} \cong[0,1)$ and doing arithmetic "modulo 1 ", at time $n$ we perturb $f$ to the map $f_{\omega_{n-1}}(x)=f\left(x+\omega_{n-1}\right)$, where $\omega_{0}, \omega_{1}, \ldots$ are IID random variables uniformly distributed in $[-\varepsilon, \varepsilon]$. Here, the noise amplitude $\varepsilon>0$ is a fixed parameter. We will consider the asymptotic dynamics of compositions of the form

$$
f_{\underline{\omega}}^{n}=f_{\omega_{n-1}} \circ \cdots \circ f_{\omega_{0}}
$$

given a sample $\underline{\omega}=\left(\omega_{0}, \omega_{1}, \ldots\right)$.

When $\varepsilon \geq 1$, random trajectories $X_{n}=f_{\underline{\omega}}^{n}\left(X_{0}\right), n \geq 1$ are essentially IID themselves; in this situation it is a straightforward exercise to check (i) uniqueness of the stationary measure for the process $\left(X_{n}\right)$ on $S^{1}$ and (ii) that the Lyapunov exponent $\lambda=$ $\lim _{n \rightarrow \infty} \frac{1}{n} \log \left|\left(f_{\underline{\omega}}^{n}\right)^{\prime}(x)\right|$ exists and is constant for every $x \in S^{1}$ and a.e. sample $\underline{\omega}$. What is more subtle is the situation when $\varepsilon \ll 1$, in which case the composition $f_{\omega}^{n}$ may develop one or more random sinks; here, for our purposes, a random sink is a stationary measure for $\left(X_{n}\right)$ with a negative Lyapunov exponent.

Random sinks can develop if, for instance, the map $f$ itself has a periodic sink $z \in S^{1}$. Indeed, it is not hard to check that the sink $z$ persists in the form of a random sink for all $\varepsilon>0$ sufficiently small (see, e.g., Section 3.1 for a worked example). On the other hand, one anticipates that sinks of $f$ of high period $N$ can be "destroyed" in the presence of a small but sufficient amount of noise, i.e., when $\varepsilon \geq \varepsilon_{N}$, where $\varepsilon_{N} \rightarrow 0$ as $N \rightarrow \infty$. As described previously, these high-period sinks are precisely those responsible for the convoluted intermingling of dynamical regimes in one-parameter families of unimodal or multimodal maps.

In an alternative perspective, given a fixed noise amplitude $\varepsilon>0$, the only sinks of $f$ which could possibly persist as random sinks for $\left(f_{\underline{\omega}}^{n}\right)$ are those of period $\leq k_{\varepsilon}:=$ $\max \left\{N: \varepsilon<\varepsilon_{N}\right\}$. A crucial point here is that, for a given map $f$, it is virtually always possible to check for sinks of period less than some given value. For these reasons, one anticipates that for a reasonably large class of $f$ as above and a given noise amplitude $\varepsilon>0$, it should be possible to determine the asymptotic chaotic regime of the corresponding random composition $f_{\omega}^{n}$ based on checkable criteria involving only finitely many iterates of the map $f$.

The present paper is a step in this direction for a model of one-parameter families of multimodal circle maps $f=f_{a}$ exhibiting strong expansion $\left(\left|f_{a}^{\prime}\right| \gg 1\right)$ away from a small neighborhood of the critical set $\left\{f_{a}^{\prime}=0\right\}$. We obtain a checkable sufficient criterion on the parameter $a$, involving only finitely many iterates of the map $f_{a}$ (in particular, precluding 
sinks of low period, as above), for deducing asymptotic chaotic behavior for the random composition $f_{\omega}^{n}$ when the noise parameter $\varepsilon$ is not too small. An appealing feature of these results is that, given $\varepsilon>0$, the criterion involves only approximately $\log \left(\varepsilon^{-1}\right)$ iterates, which grows quite slowly as $\varepsilon \rightarrow 0$.

\subsection{Statement of results}

The model. Let $S^{1}=\mathbb{R} / \mathbb{Z}$ be the unit circle, parametrized by the interval $[0,1)$. We assume throughout that $\psi: S^{1} \rightarrow \mathbb{R}$ is a $C^{2}$ function for which the following conditions hold:

(H1) The critical set $C_{\psi}^{\prime}=\left\{\hat{x} \in S^{1}: \psi^{\prime}(\hat{x})=0\right\}$ has finite cardinality.

(H2) We have $\left\{\psi^{\prime \prime}=0\right\} \cap C_{\psi}^{\prime}=\varnothing$.

We consider maps of the form

$$
f=f_{L, a}:=L \psi+a(\bmod 1),
$$

for $L>0, a \in[0,1)$, where $(\bmod 1): \mathbb{R} \rightarrow S^{1} \cong \mathbb{R} / \mathbb{Z}$ is the natural projection. Observe that for $L \gg 1$, the map $f$ is strongly expanding away from $C_{\psi}^{\prime}$.

When $\varepsilon>0$ is specified, we write $\Omega=\Omega^{\varepsilon}=([-\varepsilon, \varepsilon])^{\mathbb{Z}_{\geq 0}}$ for the sample space for our perturbations. Elements $\underline{\omega} \in \Omega$ are written $\underline{\omega}=\left(\omega_{0}, \omega_{1}, \omega_{2}, \ldots\right)$ where $\omega_{i} \in$ $[-\varepsilon, \varepsilon], i \geq 0$. With $v^{\varepsilon}$ denoting the uniform distribution on $[-\varepsilon, \varepsilon]$, we define $\mathbb{P}=\mathbb{P}^{\varepsilon}=$ $\left(v^{\varepsilon}\right)^{\otimes \mathbb{Z}_{\geq 0}}$ on $\Omega$. We write $\mathcal{F}$ for the product $\sigma$-algebra on $\Omega$ and for $n \geq 0$ we write $\widetilde{F}_{n}=\sigma\left(\omega_{0}, \omega_{1}, \ldots, \omega_{n}\right) \subset \mathcal{F}$.

When $f=f_{L, a}$ is specified, we consider random maps of the form $f_{\omega}: S^{1} \rightarrow S^{1}$, $f_{\omega}(x):=f(x+\omega)$, where it is understood implicitly that the argument for $f$ is taken (mod 1$)$. Given a sample $\underline{\omega} \in \Omega$, we have a corresponding random composition

$$
f_{\underline{\omega}}^{n}:=f_{\omega_{n-1}} \circ \cdots \circ f_{\omega_{1}} \circ f_{\omega_{0}}
$$

for $n \geq 1$.

Alternatively, we can view the random maps $f_{\underline{\omega}}^{n}$ as giving rise to a Markov chain $\left(X_{n}\right)_{n}$ on $S^{1}$ defined, for fixed initial $X_{0} \in S^{1}$, by $X_{n+1}:=f_{\omega_{n}}\left(X_{n}\right)$. The corresponding Markov transition kernel $P(\cdot, \cdot)$ is defined for $x \in S^{1}$ and Borel $B \subset S^{1}$ by

$$
P(x, B):=\mathbb{P}\left(X_{1} \in B \mid X_{0}=x\right)=v^{\varepsilon}\left\{\omega \in[-\varepsilon, \varepsilon]: f_{\omega}(x) \in B\right\} .
$$

We say that a Borel measure $\mu$ on $S^{1}$ is stationary if

$$
\mu(B)=\int_{S^{1}} P(x, B) d \mu(x)
$$

for all Borel $B \subset S^{1}$.

Results. Our results concern the following checkable, finite-time criterion $(\mathrm{H} 3)_{c, k}$ on the dynamics of $f$. For now, $c>0$ and $k \in \mathbb{N}$ are arbitrary: 
$(\mathrm{H} 3)_{c, k}$ For every $\hat{x} \in C_{\psi}^{\prime}$, we have $d\left(f^{l}(\hat{x}), C_{\psi}^{\prime}\right) \geq c$ for all $1 \leq l \leq k$.

We now state our results.

Theorem A. Let $\beta, c \in(0,1)$. Let $L>0$ be sufficiently large, depending on these constants, and assume $f=f_{L, a}$ satisfies $(\mathrm{H} 3)_{c, k}$ for some arbitrary $k \in \mathbb{N}$. Finally, assume $\varepsilon \geq L^{-(2 k+1)(1-\beta)}$. Then the random composition $f_{\underline{\omega}}^{n}$ admits a unique (hence ergodic) stationary measure $\mu$ supported on all of $S^{1}$.

Theorem B. Let $\beta, c \in(0,1)$. Let $L>0$ be sufficiently large, depending on these constants, and assume $f=f_{L, a}$ satisfies $(\mathrm{H} 3)_{c, k}$ for some arbitrary $k \in \mathbb{N}$. Finally, assume $\varepsilon \geq L^{-(2 k+1)(1-\beta)+\alpha}$ where $\alpha \geq 0$ is arbitrary. Then the Lyapunov exponent

$$
\lambda=\lim _{n} \frac{1}{n} \log \left|\left(f_{\underline{\omega}}^{n}\right)^{\prime}(x)\right|
$$

exists and is constant over $x \in S^{1}$ and $\mathbb{P}$-almost every $\underline{\omega} \in \Omega$, and satisfies the estimate

$$
\lambda \geq \lambda_{0} \log L
$$

where $\lambda_{0}=\lambda_{0}(\alpha, k):=\min \left\{\frac{\alpha}{k+1}, \frac{1}{10}\right\}$.

Theorems A, B are approximately sharp, in the sense that $(\mathrm{H} 3)_{c, k}$ is compatible with the formation of sinks of period $k+1$, while such sinks persist under random perturbations $\varepsilon \leq C L^{-(2 k+1)}$ where $C>0$ is a constant. See Proposition 3.1 for more information.

A satisfying feature of our results is that, for fixed sufficiently large $L$ and any given $\varepsilon>0$, to deduce a large positive exponent for $f=f_{L, a}$ requires validating condition $(\mathrm{H} 3)_{c, k}$ with $k=k_{\varepsilon} \approx \log \left(\varepsilon^{-1}\right)$. The value of $k_{\varepsilon}$ grows only logarithmically with $\varepsilon^{-1}$, which means that even for quite small $\varepsilon>0$, Theorems $\mathrm{A}, \mathrm{B}$ are already valid when $(\mathrm{H} 3)_{c, k}$ is verified for a relatively small value of $k$.

Prior work. There is a substantial and growing literature on random dynamical systems in low dimensions: we recall below some of the literature on random dynamical systems closest to the present paper, i.e., dealing with random maps having strong expansion mixed with some contraction in phase space.

Lian and Stenlund ([33]) consider random perturbations of predominantly expanding (expanding on most of phase space with a small exceptional set) multimodal maps, more or less equivalent to the model in the present paper. They prove that for large enough noise amplitudes, the random system has a unique ergodic stationary measure and a positive Lyapunov exponent. They develop a similar result with smaller noise amplitude assuming a "one time-step" condition on the dynamics, essentially equivalent to $(\mathrm{H} 3)_{c, 1}$ in our paper. Because we deal with higher-iterate dynamical assumptions, the perturbations we may consider are substantially smaller than those in [33].

Stenlund and Sulku ([45]) obtain exponential loss of memory for IID compositions $T^{n}=T_{n} \circ \cdots \circ T_{1}$ of random circle maps which are "expanding on average": contractive behavior (inf $\left|T^{\prime}\right| \approx 0$ ) can appear with positive probability, but the random variable 
$\inf \left|T^{\prime}\right|$ satisfies a moment condition. The random maps we consider in the present paper always have critical points, and so do not satisfy the conditions of [45].

In joint work with the first author ([10-12]), random perturbations of a model of "predominantly hyperbolic" two-dimensional maps are considered. The paper [11] considers a volume-preserving model encompassing the Chirikov standard map, [12] considers a dissipative (volume-compressing) model of maps having qualitative similarities to the Hénon maps, while the more recent [10] considers systems consisting of arbitrarily many coupled volume-preserving maps. Chaotic properties of the deterministic dynamics in each case are anticipated to hold on large subsets of parameter space, but rigorous verification is largely beyond the scope of current studies. What [10-12] show is that sufficiently large random perturbations have the effect of "unlocking" the hyperbolicity of these systems (positive Lyapunov exponent proportional to the Lebesgue average $\int \log \left\|d F_{x}\right\| d x$, estimate of decay of correlations). A different but related analysis is carried out in the paper of Ledrappier, Simó, Shub, and Wilkinson ([31]), which considers IID perturbations applied to a twist map on the sphere.

Additionally, [11, 12] allow smaller random perturbations on assuming a checkable condition involving the first several iterates of the deterministic map, consistent with the finite-time checkable criterion given in the present paper.

To reiterate, the papers [10-12,31,33,45] are emphasized because they deal with random perturbations of maps for which very little is assumed: in these studies, the randomness itself is leveraged in a crucial way to "shake loose" hyperbolicity. Other works examine random compositions of maps with known "good" asymptotic behavior: by way of example, we mention works on smooth ([3,43]) and piecewise ([14]) expanding maps, maps with a neutral fixed point ([1]). This also includes works on the problem of stochastic stability: Under what conditions do properties of a given deterministic system persist under small random perturbations? There are many works in this important direction, for example, work on small random perturbations of Axiom A systems ([29,50]), unimodal maps under an (uncheckable) infinite-time condition ([2,8,27]) and stochastic stability for Hénon attractors ([7]). We also acknowledge the related problem of statistical stability, e.g., how long-time statistics of a dynamical system change within a parametrized family: see, e.g., the review [44].

The study of deterministic one-dimensional maps with critical points (unimodal or multimodal) has a long history, a small part of which we recall here. Naturally we inherit and use some of the ideas developed in this literature. Indeed, our criterion $(\mathrm{H} 3)_{c, k}$ is a checkable, finite-time version of various criteria on postcritical orbits of unimodal and multimodal maps as used by, e.g., Misiurewicz ([38]), Jakobson ([26]), Collet and Eckmann ([16]) and Benedicks and Carleson ([4]). We note as well the more expository account by Wang and Young ([48]), which we found remarkably helpful in preparing this work. There are also by now several works attempting to quantify the set of parameters for the quadratic map family at which various dynamical regimes are observed $([20,23,36,46])$. Also related to our finite-time checkable criteria are frameworks attempt- 
ing to understand dynamical properties at "finite resolution" ([19,35]) or along finite, bounded timescales ([9]).

Potential future directions. A natural future direction is to study small random perturbations of the Hénon map and related models, in the hope that one can derive checkable finite-time conditions for a positive Lyapunov exponent for the corresponding stationary measure. ${ }^{1}$ This has been carried out for the standard map and a family of dissipative mappings with "Hénon flavor" using finite-time conditions amounting to three steps of the deterministic dynamics in the previous work [11,12]; the goal of future work would be to go beyond this and derive a succession of stronger finite-time conditions allowing for smaller noise amplitudes. On the other hand, studying Lyapunov exponents for models of this kind this is likely to entail several fundamental challenges not addressed in the present manuscript, e.g., coping with the fact that one must now track tangent directions as well as the location in phase space.

Organization of the paper. In Section 2 we derive elementary properties of our model used throughout the paper, especially the notion of bound period defined in Section 2.2. In Section 3.1 we discuss the possible formation of sinks of period $k+1$ under the condition $(\mathrm{H} 3)_{c, k}$, verifying the relative sharpness of Theorems A, B; ergodicity as in Theorem A is then proved in Section 3.2. The material in Section 3 depends on Section 2 but is otherwise logically isolated from the rest of the manuscript. The proof of Theorem B occupies the remainder of the paper, Sections 4-6.

\section{Notation.}

- Throughout, we parametrize $S^{1}$ by the half-open interval $[0,1) \cong \mathbb{R} / \mathbb{Z}$. For $s \in \mathbb{R}$, we write $s(\bmod 1)$ for the projection of $s$ to $[0,1) \cong \mathbb{R} / \mathbb{Z}$ modulo 1 .

- We define the lift $\tilde{f}: S^{1} \rightarrow \mathbb{R}$ of $f$ by $\tilde{f}(x)=L \psi(x)+a$ (i.e., without projecting $(\bmod 1)$ to $\left.S^{1}\right)$. We regard $\tilde{f}$ as a map $\mathbb{R} \rightarrow \mathbb{R}$ by extending the domain periodically to all of $\mathbb{R}$. We write $\tilde{f}_{\omega}(x)=\tilde{f}(x+\omega)$. We define the corresponding Markov process $\left(\tilde{X}_{n}\right)_{n}$ on $\mathbb{R}$ by setting $\tilde{X}_{n+1}=\tilde{f}_{\omega_{n}}\left(\tilde{X}_{n}\right)$.

- We write $d(\cdot, \cdot)$ for the metric induced on $S^{1}$ via the identification with $\mathbb{R} / \mathbb{Z} \cong$ $[0,1)$. Note that in our parametrization, we have the identity $d(x, y)=\min \{|x-y|$, $|x-y \pm 1|\}$. For a set $A \subset S^{1}$, we write $\mathcal{N}_{\varepsilon}(A)$ for the $\varepsilon$-neighborhood of $A$ in the metric $d$.

- For a point $x \in S^{1}$ and a set $A \subset S^{1}$, we define the minimal distance $d(x, A)=$ $\inf _{a \in A} d(x, a)$. Further, for sets $A, B \subset S^{1}$, we define $d(A, B)=\inf _{a \in A} d(a, B)=$ $\inf _{a \in A, b \in B} d(a, b)$.

- Given a set $A \subset S^{1}$ or $\mathbb{R}$ and $z \in S^{1}$ or $\mathbb{R}$, we write $A-z=\{a-z: a \in A\}$ for the set $A$ shifted by $z$.

\footnotetext{
${ }^{1}$ For random systems with absolutely continuous stationary measures, a positivity of Lyapunov exponent implies existence of "random strange attractors" analogous to those for deterministic systems $([13,32])$.
} 
- Given a partition $\zeta$ of $S^{1}$ (resp. $\mathbb{R}$ ) and a set $A \subset S^{1}$ (resp. $A \subset \mathbb{R}$ ), we write $\left.\zeta\right|_{A}$ for the partition on $A$ consisting of atoms of the form $C \cap A, C \in \zeta, C \cap A \neq \emptyset$.

- When it is clear from context, we write $\mathbb{E}$ for the expectation with respect to $\mathbb{P}$.

\section{Preliminaries: predominant expansion and bound periods}

Bound periods: a heuristic. Consider the dynamics of a smooth unimodal or multimodal map $f: S^{1} \rightarrow S^{1}$. In the pursuit of finding maps $f$ accumulating a positive Lyapunov exponent, the main obstruction is the formation of sinks, and so a natural assumption to make is that the postcritical orbits $f^{n} \hat{x}, \hat{x} \in\left\{f^{\prime}=0\right\}, n \geq 1$ remain far enough away from $\left\{\left|f^{\prime}\right| \leq 1\right\}$ so that $\left|\left(f^{n}\right)^{\prime}(f \hat{x})\right| \gtrsim e^{n \alpha}$ for some $\alpha>0$.

If, for some $x \in S^{1}$, the orbit $\left(f^{n} x\right)_{n}$ reaches a small neighborhood of some $\hat{x} \in$ $\left\{f^{\prime}=0\right\}$ at time $t$, then the subsequent iterates $f^{t+i} x$ will closely shadow $f^{i} \hat{x}$ for $i \leq$ $p=p\left(d\left(f^{t} x, \hat{x}\right)\right)$. The time interval $[t+1, t+p]$ is referred to as the bound period for $x$ at time $t$. As we assumed expansion along the postcritical orbit $\left(f^{i} \hat{x}\right)_{i \geq 1}$, one anticipates that the derivative growth $\left(f^{p}\right)^{\prime}\left(f^{t+1} x\right)$ accumulated along the bound period will balance out the derivative "damage" due to $f^{\prime}\left(f^{t} x\right)$ (possibly $\ll 1$ when $f^{t} x, \hat{x}$ are quite close), so that, for instance, $\left(f^{p+1}\right)^{\prime}\left(f^{t} x\right) \sim e^{(p+1) \alpha^{\prime}}$ holds for some $\alpha^{\prime}<\alpha$.

This is a rough summary of a mechanism by which one-dimensional maps with critical points (unimodal and multimodal) can accumulate a positive Lyapunov exponent for typical trajectories. For an exposition of this method, see [48].

Our aim in Section 2 is to apply a variation of this idea to our model: the condition $(\mathrm{H} 3)_{c, k}$ involves the first $k$ iterates of postcritical trajectories, and so bound periods of length up to $k$ are available to recover derivative growth. In Section 2.1 we carry out some essential preliminaries used in the rest of the paper, and in Section 2.2 we will discuss bound periods for our random compositions.

\subsection{Preliminaries}

2.1.1. The basic setup. We fix, below and throughout the paper, a function $\psi: S^{1} \rightarrow \mathbb{R}$ satisfying (H1) and (H2), as well as parameters $c \in(0,1), \beta \in\left(0, \frac{1}{100}\right)$ (restricting to $\beta$ in this range incurs no loss of generality). Moreover, we implicitly fix the parameter $L>0$, and are allowed to take it sufficiently large depending on $c, \beta$ and the function $\psi$.

On rescaling the function $\psi$ in relation to the parameter $L$, we will assume going forward that the following condition holds in addition to $(\mathrm{H} 1)-(\mathrm{H} 2)$.

(H4) We have $\left\|\psi^{\prime}\right\|_{C^{0}},\left\|\psi^{\prime \prime}\right\|_{C^{0}} \leq \frac{1}{10}$.

Separately (i.e., independently of $L$ ), $k \in \mathbb{N}$ is fixed, and a parameter $a \in[0,1)$ is fixed for which $(\mathrm{H} 3)_{c, k}$ holds for the mapping $f=f_{L, a}:=L \psi+a(\bmod 1)$. Finally, we fix a parameter $\varepsilon>0$, on which constraints (depending on all the previous parameters) will be made as we go along. 
2.1.2. Partition of phase space. Conditions $(\mathrm{H} 1)-(\mathrm{H} 2)$ imply that there is a constant $K_{1}=K_{1}(\psi)>0$ with the property that for any $x \in S^{1}$,

$$
\left|\psi^{\prime}(x)\right| \geq K_{1} d\left(x, C_{\psi}^{\prime}\right) .
$$

We use (1) repeatedly, often without mention. For $\eta<0$, we define

$$
B(\eta)=\left\{x \in S^{1}: d\left(x, C_{\psi}^{\prime}\right) \leq K_{1}^{-1} L^{\eta}\right\}
$$

It is clear that for $x \notin B(\eta)$, we have $\left|f^{\prime}(x)\right| \geq L^{\eta+1}$, while $B(\eta)$ is the union of $\# C_{\psi}^{\prime}$ intervals of length $\sim L^{\eta}$ each.

Define the partition $S^{1}=\mathscr{E} \cup \mathcal{I} \cup \mathscr{B}$, where

$$
\mathcal{E}=S^{1} \backslash B(-\beta), \quad \mathcal{I}=B(-\beta) \backslash B\left(-\frac{1}{2}-\beta\right), \quad \mathscr{B}=B\left(-\frac{1}{2}-\beta\right) .
$$

We have, then, that

$$
\left|f^{\prime}\right| \boldsymbol{E} \mid \geq L^{1-\beta} \quad \text { and } \quad\left|f^{\prime}\right| \mathcal{I} \mid \geq L^{\frac{1}{2}-\beta} .
$$

Similar estimates apply to $f_{\omega}^{\prime}$ on the shifted sets $\mathcal{E}_{\omega}:=\mathcal{E}-\omega, \mathcal{I}_{\omega}:=\mathcal{I}-\omega$ for $\omega \in$ $[-\varepsilon, \varepsilon]$.

Observe that $\left|f^{\prime}\right|_{\mathscr{B}} \mid$ can be arbitrarily small. To address this, we subdivide $\mathscr{B}=$ $\bigcup_{l=1}^{k} \mathscr{B}^{l}$ in the following way: set

$$
\mathscr{B}^{k}=B\left(-\frac{k}{2}-\beta\right)
$$

and for $1 \leq l<k$,

$$
\mathcal{B}^{l}=B\left(-\frac{l}{2}-\beta\right) \backslash B\left(-\frac{l+1}{2}-\beta\right) .
$$

Notice that the definition above is consistent with the identification $\mathcal{I}=\mathscr{B}^{0}$. We also use the notation $\mathscr{B}_{\omega}^{l}:=\mathscr{B}^{l}-\omega$ for $\omega \in[-\varepsilon, \varepsilon]$. Using (1), one checks that

$$
\left|f_{\omega}^{\prime}\right|_{B_{\omega}^{l}} \mid \geq L^{-\frac{l-1}{2}-\beta} \quad \text { for } 1 \leq l<k,
$$

while on $\mathscr{B}_{\omega}^{k}$ we have no lower bound on $\left|f_{\omega}^{\prime}\right|$.

The partitions $S^{1}=\mathcal{E} \cup \mathcal{I} \cup \mathcal{B}=\mathscr{E} \cup \mathcal{I} \cup \mathscr{B}_{1} \cup \cdots \cup \mathscr{B}^{k}$ are used repeatedly throughout the paper. We will abuse notation and regard these as partitions of $\mathbb{R}$ as well, extended by periodicity via the parametrization $S^{1} \cong[0,1) \cong \mathbb{R} / \mathbb{Z}$.

\subsection{Bound periods}

The following lemma confirms that a random orbit $\left(f_{\omega}^{i} x\right)$, initiated at $x \in \mathscr{B}^{l}, 1 \leq l \leq k$ will closely shadow a postcritical orbit $\left(f^{i} \hat{x}\right)$ for $l$ steps, i.e., will have a bound period of length $l$.

In Lemma 2.1 below we do not assume $(\mathrm{H} 3)_{c, k}$. 
Lemma 2.1. Let $L$ be sufficiently large, and let $k \in \mathbb{N}$ be arbitrary. Assume that

$$
\varepsilon<L^{-\max \left\{k-1, \frac{1}{2}\right\}-\beta} .
$$

Then we have the following. Let $1 \leq l \leq k$ and fix an arbitrary sample $\underline{\omega} \in \Omega$. Let $J_{0}$ be any connected component of $B\left(-\frac{l+\beta}{2}\right)$ and let $\hat{x}=C_{\psi}^{\prime} \cap J$ be the (unique) critical point contained in $J_{0}$.

Then, for all $1 \leq i \leq l$, we have

$$
f_{\underline{\omega}}^{i}\left(J_{0}\right) \subset \mathcal{N}_{L^{-\beta / 2}}\left(f^{i} \hat{x}\right) .
$$

The reason for the upper bound (3) is that if the perturbation amplitude $\varepsilon$ is too large, then $\left.f_{\underline{\omega}}^{i}\right|_{\mathcal{B}_{\omega_{0}}^{l}}$ may diverge from $f^{i} \hat{x}$ for some $i<k$, thereby spoiling the corresponding bound periods.

From Lemma 2.1 and noting $\mathscr{B}^{l} \subset B\left(-\frac{l+\beta}{2}\right)$, it is straightforward to check that if $L$ is sufficiently large and $f=f_{a}$ satisfies $(\mathrm{H} 3)_{c, k}$, then $f^{i} \hat{x}$ is well inside $\boldsymbol{G}$ for $1 \leq i \leq k$. It follows that for any $1 \leq l \leq k$ and $x \in \mathscr{B}_{\omega_{0}}^{l}$, we have $f_{\underline{\omega}}^{i}(x) \in \mathcal{G}$ for all $1 \leq i \leq l$, and the derivative estimate

$$
\left|\left(f_{\theta \underline{\omega}}^{l}\right)^{\prime}\left(f_{\omega_{0}} x\right)\right| \geq L^{l(1-\beta)} .
$$

Moreover, if $1 \leq l<k$ then we have $\left|\left(f_{\omega_{0}}\right)^{\prime}(x)\right| \geq L^{1-\frac{l+1}{2}-\beta}$, hence

$$
\left|\left(f_{\underline{\omega}}^{l+1}\right)^{\prime}(x)\right| \geq L^{(l+1)\left(\frac{1}{2}-\beta\right)} .
$$

For the purposes of the preceding paragraph, it suffices to take $L$ large enough so that $L^{\beta} \gg 2 /\left(c K_{1}\right)$; note in particular that $L$ does not depend on $k$.

Proof of Lemma 2.1. In the following proof, the lift $\tilde{f}: S^{1} \rightarrow \mathbb{R}$ of $f$ is defined by $\tilde{f}(x)=$ $L \psi(x)+a$, i.e., leaving out the " $(\bmod 1)$ " in the definition of $f$. We extend the domain of $\tilde{f}$ to all of $\mathbb{R}$ by periodicity.

Without loss, we regard $J_{0}$ as an interval in $\mathbb{R}$. Let $\hat{x} \in C_{\psi}^{\prime} \cap J_{0}$ be the (unique) critical point in $J_{0}$. Define $I_{0}=\mathcal{N}_{\varepsilon}\left(J_{0}\right)$ and inductively set $J_{i+1}=\tilde{f}\left(I_{i}\right), I_{i+1}=\mathcal{N}_{\varepsilon}\left(J_{i+1}\right)$. Since $f^{i} \hat{x} \in J_{i}$ for all $i$, it suffices to show $\operatorname{Len}\left(J_{i}\right) \leq L^{-\beta / 2}$ for all $1 \leq i \leq l$.

To start, decompose $I_{0}=I_{0}^{-} \cup I_{0}^{+}$where $I_{0}^{-}=\left[\hat{x}-\varepsilon-K_{1}^{-1} L^{-\frac{l+\beta}{2}}, \hat{x}\right), I_{0}^{+}=$ $\left[\hat{x}, \hat{x}+\varepsilon+K_{1}^{-1} L^{-\frac{l+\beta}{2}}\right]$. Noting that the images $\tilde{f}\left(I_{0}^{-}\right), \tilde{f}\left(I_{0}^{+}\right)$share the left (resp. right) endpoint $\tilde{f}(\hat{x})$ if $f^{\prime \prime}(\hat{x})>0$ (resp. $\left.f^{\prime \prime}(\hat{x})<0\right)$, we have the estimate

$$
\begin{aligned}
\operatorname{Len}\left(J_{1}\right) & \leq \max \left\{\tilde{f}\left(I_{0}^{+}\right), \tilde{f}\left(I_{0}^{-}\right)\right\} \leq \frac{1}{2} L\left\|\psi^{\prime \prime}\right\|_{C^{0}} \cdot\left(\varepsilon+K_{1}^{-1} L^{-\frac{l+\beta}{2}}\right)^{2} \\
& \leq L \max \left\{\varepsilon, \operatorname{Len}\left(J_{0}\right)\right\}^{2}
\end{aligned}
$$

using (H4) in the last step. For each $i>1$, we estimate

$$
\operatorname{Len}\left(J_{i}\right)=\operatorname{Len}\left(\tilde{f}\left(I_{i-1}\right)\right) \leq L\left\|\psi^{\prime}\right\|_{C^{0}} \operatorname{Len}\left(I_{i-1}\right) \leq L \max \left\{\varepsilon, \operatorname{Len}\left(J_{i-1}\right)\right\}
$$


by estimating $\operatorname{Len}\left(I_{i-1}\right) \leq 2 \varepsilon+\operatorname{Len}\left(J_{i-1}\right) \leq 3 \max \left\{\varepsilon\right.$, Len $\left.\left(J_{i-1}\right)\right\}$ and using (H4). Bootstrapping, we conclude

$$
\operatorname{Len}\left(J_{i}\right) \leq L^{i-1} \max \left\{\varepsilon, \operatorname{Len}\left(J_{1}\right)\right\} \leq \max \left\{L^{i-1} \varepsilon, L^{i} \varepsilon^{2}, L^{i} \operatorname{Len}\left(J_{0}\right)^{2}\right\} .
$$

The first two terms are $<L^{-\beta}$ by (3) for all $i \leq k$. For $i \leq l$, the third term is $\leq L^{i} \cdot 4 K_{1}^{-2} L^{-l-\beta} \leq L^{-\beta / 2}$. This completes the proof.

\section{Ergodicity}

In Section 3.1 we prove Proposition 3.1, which confirms the sharpness of Theorems A, $\mathrm{B}$ in the following sense. To start, condition $(\mathrm{H} 3)_{c, k}$ for the map $f=f_{a}$ is compatible with the formation of a sink of period $k+1$. For all $\varepsilon>0$ sufficiently small, such sinks persist as random sinks for the random compositions $\left(f_{\underline{\omega}}^{n}\right)$, i.e., stationary measures for the Markov chain $\left(X_{n}\right)_{n}$ admitting a negative Lyapunov exponent. In Proposition 3.1 we make this quantitative by exhibiting a scenario when $f=f_{a}$ (i) satisfies (H3) $)_{, k}$, (ii) admits a sink of period $k+1$ and (iii) the random composition $\left(f_{\underline{\omega}}^{n}\right)$ admits a random sink for all $\varepsilon \lesssim L^{-(2 k+1)}$. This upper bound for $\varepsilon$ approximately matches the upper bound in Theorems A, B, confirming the view that these results are sharp.

Having established this, in Section 3.2 we proceed with the proof of Theorem A. We note that in terms of logical dependence, Section 3 depends on Section 2 and is otherwise independent of the remainder of the paper (Sections 4-6).

\subsection{Sinks}

Let us take on the assumptions made for the map $f=f_{L, a}$ as in Section 2.1.1, except that for Proposition 3.1 we need not assume $(\mathrm{H} 3)_{c, k}$ holds. Observe, however, that the hypothesis of Proposition 3.1, i.e., the existence of a sink of period $k+1$ for $f=f_{L, a}$, is entirely compatible with $(\mathrm{H} 3)_{c, k}$.

Proposition 3.1. For all L sufficiently large, depending only on $\psi$, we have the following. Let $k \in \mathbb{N}$ be arbitrary, and assume $f=f_{L, a}$ has the property that $f^{k+1} \hat{x}=\hat{x}$ for some $\hat{x} \in C_{\psi}^{\prime}$. Then, for any $\varepsilon \leq \frac{1}{49} L^{-(2 k+1)}$, we have that the random composition $f_{\underline{\omega}}^{n}$ admits a stationary measure $\mu$ for which

(a) the support of $\mu, \operatorname{Supp}(\mu)$, is contained in a $\frac{1}{7} L^{-(k+1)}$-neighborhood of the orbit $\hat{x}, f \hat{x}, \ldots, f^{k} \hat{x}$ (in particular, Supp $\mu \subsetneq S^{1}$ );

(b) $\lambda_{1}(\mu)<0$.

Proof. We will show that there is a neighborhood $U$ of $\hat{x}$ such that for a.e. sample $\underline{\omega} \in \Omega$,

(i) $f_{\underline{\omega}}^{k+1}(U) \subset U$;

(ii) $\left|\left(f_{\underline{\omega}}^{k+1}\right)^{\prime}(x)\right|<\frac{1}{2}$ for all $x \in U$. 
By standard arguments, (i)-(ii) imply the existence of a stationary measure $\mu$ with Lyapunov exponent $\lambda(\mu) \leq-\log 2 /(k+1)<0$ supported in $\left\{f_{\underline{\omega}}^{i} x: x \in U, \underline{\omega} \in \Omega\right.$, $0 \leq i \leq k\}$. At the end, we will estimate the size of this support.

Let $\gamma \in(0,1)$ be a constant, to be taken sufficiently small below, and throughout assume that $\varepsilon \leq \gamma L^{-(2 k+1)}$. Set $U$ to be the closed neighborhood of $\hat{x}$ of radius $r_{U}=$ $\sqrt{\gamma} L^{-(k+1)}$. We estimate

$$
\begin{aligned}
\sup _{z \in U}\left|\left(f_{\underline{\omega}}^{i}\right)^{\prime}(z)\right| \leq\left\|f^{\prime}\right\|_{C^{0}}^{i-1} \cdot\left(\varepsilon+\sqrt{\gamma} L^{-(k+1)}\right) \cdot\left\|f^{\prime \prime}\right\|_{C^{0}} & \leq L^{i} \cdot 2 \sqrt{\gamma} L^{-(k+1)} \\
& \leq 2 \sqrt{\gamma} L^{i-(k+1)},
\end{aligned}
$$

having used the elementary bound $\left|f_{\omega}(z)\right| \leq|z+\omega-\hat{x}| \cdot\left\|f^{\prime \prime}\right\|_{C^{0}} \leq L|z+\omega-\hat{x}|$ for $z$ near $\hat{x}$. In particular, at $i=k+1$ we have

$$
\left|\left(f_{\underline{\omega}}^{k+1}\right)^{\prime}\right|_{U} \mid \leq 2 \sqrt{\gamma},
$$

hence $U$ maps to an interval $f_{\underline{\omega}}^{k+1}(U)$ of length $\left|f_{\underline{\omega}}^{k+1}(U)\right| \leq 2 \sqrt{\gamma} \cdot|U|=4 \sqrt{\gamma} \cdot r_{U}$.

Let us now estimate $d\left(\hat{x}, \bar{f}_{\omega}^{k+1}(\hat{x})\right)$. For simplicity, we pass to the lifts $\tilde{f}, \tilde{f}_{\omega}$ : write $\hat{x}^{i}=\tilde{f}^{i} \hat{x}, \hat{x}_{\underline{\omega}}^{i}=\tilde{f}_{\underline{\omega}}^{i} \hat{x}$ for $0 \leq \underline{i} \leq k+1$. To start,

$$
\left|\hat{x}^{1}-\hat{x}_{\underline{\omega}}^{1}\right|=\left|\tilde{f}(\hat{x})-\tilde{f}\left(\hat{x}+\omega_{0}\right)\right| \leq \varepsilon \cdot \sup _{d(z, \hat{x}) \leq \varepsilon}\left|f^{\prime}(z)\right| \leq \varepsilon^{2} L .
$$

Next, for $i>0$,

$$
\left|\hat{x}^{i+1}-\hat{x}_{\underline{\omega}}^{i+1}\right|=\left|\tilde{f}\left(\hat{x}^{i}\right)-\tilde{f}\left(\hat{x}_{\underline{\omega}}^{i}+\omega_{i}\right)\right| \leq L\left(\varepsilon+\left|\hat{x}^{i}-\hat{x}_{\underline{\omega}}^{i}\right|\right) .
$$

Collecting, we obtain

$$
\begin{aligned}
d\left(\hat{x}, f_{\underline{\omega}}^{k+1}(\hat{x})\right) \leq\left|\hat{x}-\hat{x}_{\underline{\omega}}^{k+1}\right| & \leq\left(L+L^{2}+\cdots+L^{k}\right) \varepsilon+L^{k+1} \varepsilon^{2} \\
& \leq 2 L^{k} \varepsilon+L^{k+1} \varepsilon^{2} \leq 3 \gamma L^{-(k+1)}
\end{aligned}
$$

here having assumed $L>2$. We deduce

$$
d\left(\hat{x}, f_{\underline{\omega}}^{k+1}(\hat{x})\right) \leq 3 \sqrt{\gamma} \cdot r_{U} .
$$

It is easy to check that the same bound $d\left(\hat{x}^{i}, f_{\underline{\omega}}^{i}(\hat{x})\right) \leq 3 \sqrt{\gamma} \cdot r_{U}$ holds for any $0 \leq i \leq k$ as well.

To conclude, for (i) it suffices (see (4)) to take $\gamma \leq \frac{1}{16}$. For (ii) we estimate as follows for $z \in U$ :

$$
d\left(f_{\underline{\omega}}^{k+1}(z), \hat{x}\right) \leq d\left(\hat{x}, f_{\underline{\omega}}^{k+1}(\hat{x})\right)+\left|f_{\underline{\omega}}^{k+1}(U)\right| \leq 7 \sqrt{\gamma} \cdot r_{U} .
$$

We conclude that $f_{\underline{\omega}}^{k+1}(U) \subset U$ almost surely as long as $\gamma \leq \frac{1}{49}$.

Finally, to estimate the support of $\mu$ it suffices to repeat estimate (5) with $f_{\underline{\omega}}^{i}(z), z \in U$ replacing $f_{\underline{\omega}}^{k+1}(z)$. We conclude that $\mu$ is supported in the $7 \sqrt{\gamma} \cdot r_{U}$-neighborhood of the periodic sink $\left\{f^{i} \hat{x}\right\}_{0 \leq i \leq k}$. 


\subsection{Ergodicity}

As already seen in the proofs of Lemma 2.1 and Proposition 3.1, the noise amplitude $\varepsilon$ is amplified by the strong expansion $L \gg 1$ exhibited by $f=f_{L, a}$. Each of these results depended on the noise being small enough to control this amplification. Quite to the contrary, in Section 3.2 we will take advantage of this amplification to show that our process $\left(X_{n}\right)$ explores all of phase space $S^{1}$ with some positive probability. The amplification of noise by expansion is a core motif in this paper, and one that we will return to in Sections 5-6.

Before proceeding to the proof of Theorem A, let us establish the setting and a brief reduction. Throughout, we assume the setup for $f=f_{L, a}$ in Section 2.1.1, including $(\mathrm{H} 3)_{c, k}$.

Reductions. We first argue that without loss of generality, in the hypotheses of Theorem A we may assume that $\varepsilon, k$ are such that the upper bound in (3) is satisfied, so that Lemma 2.1 applies. To justify this, consider the following alternative cases:
(a) $L^{-(k-1)} \leq \varepsilon<L^{-1}$
(b) $L^{-1} \leq \varepsilon<L^{-1 / 2}$,
(c) $\varepsilon \geq L^{-1 / 2}$

For (a), let $k^{\prime} \in \mathbb{N}$ be such that $L^{-k^{\prime}} \leq \varepsilon<L^{-\left(k^{\prime}-1\right)}$. Clearly $k^{\prime}<k$, hence $(\mathrm{H} 3)_{c, k}$ implies (H3) $c, k^{\prime}$, while $\varepsilon \geq L^{-k^{\prime}} \geq L^{-\left(2 k^{\prime}+1\right)(1-\beta)+\beta}$. So, it makes no difference to replace $k$ with $k^{\prime}$ and proceed as before. In case (b), we can replace $k$ with 1 and proceed as before. Finally, Theorem A in case (c) is a simple exercise left to the reader - see also [33, Theorem 1], where ergodicity as in Theorem A is proved for $\varepsilon \gtrsim L^{-1}$ for a very similar model of multimodal circle maps.

In addition, on shrinking the parameter $\beta$ we will assume the slightly stronger hypothesis

$$
\varepsilon \geq L^{-(2 k+1)(1-\beta)+\beta}
$$

on the noise parameter $\varepsilon$. In relation to Theorem A, this incurs no loss of generality.

Notation. Given an initial $X_{0} \in S^{1}$, we write $X_{n}=f_{\omega}^{n}\left(X_{0}\right)$ for the Markov chain evaluated at the sample $\underline{\omega} \in \Omega$ (notation as in Section 1.1). We write $\mathbb{P}_{X_{0}}$ for the law of $X_{n}$ conditioned on the value of $X_{0} \in S^{1}$. Moreover, for $n, m \geq 0$, random variables $Z_{1}, Z_{2}, \ldots, Z_{m}: \Omega \rightarrow \mathbb{R}$, and $X_{0} \in S^{1}$, we write

$$
P^{n}\left(X_{0}, \cdot \mid\left\{Z_{j}, 1 \leq j \leq m\right\}\right)=\mathbb{P}_{X_{0}}\left(X_{n} \in \cdot \mid \sigma\left(Z_{1}, \ldots, Z_{m}\right)\right)
$$

for the law of $X_{n}$ conditioned on $\sigma\left(Z_{1}, Z_{2}, \ldots, Z_{m}\right)$.

With the setup and reduction established, we now turn to the proof of Theorem A. We break this up into two parts, Propositions 3.2 and 3.3 below.

Proposition 3.2. There exist $N \in \mathbb{N}, c>0$ with the property that for any sample $\underline{\omega}$ and any $X_{0} \in \mathscr{B}_{\omega_{0}}^{k}$, we have $P^{N}\left(X_{0}, \cdot \mid\left\{\omega_{i}, 0 \leq i \leq N, i \neq 1\right\}\right) \geq c \operatorname{Leb}(\cdot)$.

What this means is that random trajectories initiated in $\mathscr{B}^{k}$ reach all of $S^{1}$ with some positive probability. Note that in Proposition 3.2, we randomize only in $\omega_{1}$. One reason is 
that since $X_{0} \in \mathcal{B}_{\omega_{0}}^{k}$, we have that $X_{1}, X_{2}, \ldots, X_{k}$ experience a bound period of length $k$, and so $\omega_{1}$ is the only perturbation which experiences the full $k$ steps of expansion guaranteed by Lemma 2.1. Meanwhile, it is technically more convenient to work with one perturbation $\omega_{i}$ at a time.

By Proposition 3.2, it suffices to check that almost every trajectory enters $\mathscr{B}^{k}$ after a finite time. Define the stopping time

$$
T:=\min \left\{i \geq 0: X_{i} \in \mathcal{B}_{\omega_{i}}^{k}\right\} .
$$

Proposition 3.3. Assume the hypotheses of Theorem A. Then there exists $\widehat{N} \in \mathbb{N}$ such that for any $X_{0} \in S^{1}$, we have $\mathbb{P}_{X_{0}}(T \leq \widehat{N})>0$.

Proof of Theorem A assuming Propositions 3.2, 3.3. Observe that ergodic measures $\mu$ (1) exist by a standard tightness argument and (2) automatically inherit absolute continuity with respect to Lebesgue on $S^{1}$ from the same property for our random perturbations $\omega_{i}, i \geq 0$. So, to conclude uniqueness, it suffices to check that for all $X_{0} \in S^{1}, P^{M}\left(X_{0}, \cdot\right)$ is supported on all of $S^{1}$ (i.e., assigns positive mass to all open intervals) for some $M=M\left(X_{0}\right) \in \mathbb{N}$. For more details, see, e.g., the characterization of ergodicity for stationary measures of random dynamical systems in [28, Lemma 2.4 on p. 19].

To complete the proof, fix $X_{0} \in S^{1}$ and let $n \leq \hat{N}$ be such that $\mathbb{P}_{X_{0}}(T=n)>0$. Then, for any interval $J \subset S^{1}$ with nonempty interior,

$$
\begin{aligned}
P^{n+N}\left(X_{0}, J\right) & =\mathbb{E}\left(P^{N}\left(X_{n}, J \mid\left\{\omega_{i}\right\}_{0 \leq i \leq n+N, i \neq n+1}\right)\right) \\
& \geq \mathbb{E}\left(\chi_{T=n} \cdot P^{N}\left(X_{n}, J \mid\left\{\omega_{i}\right\}_{0 \leq i \leq n+N, i \neq n+1}\right)\right) \\
& \geq \mathbb{E}\left(\chi_{T=n} \cdot c \operatorname{Leb}(I)\right)=c \cdot \mathbb{P}_{X_{0}}(T=n) \cdot \operatorname{Leb}(I)>0 .
\end{aligned}
$$

Here, $\mathbb{E}_{X_{0}}$ refers to the expectation conditioned on the value of $X_{0}$.

This completes the proof. It remains to check Propositions 3.2, 3.3.

In the remainder of Section 3, we prove Propositions 3.3, 3.2, in that order. With the above setup assumed, we hereafter fix $\varepsilon \in\left[L^{-(2 k+1)(1-\beta)+\beta}, L^{-\max \left\{k-1, \frac{1}{2}\right\}}\right]$.

3.2.1. Constructions and a preliminary lemma. Define $\mathcal{R}$ to be the partition of $S^{1}$ into the connected components of the sets $\mathcal{E}, \mathcal{I}=\mathscr{B}^{0}, \mathscr{B}^{1}, \ldots, \mathscr{B}^{k}$. For $\omega \in[-\varepsilon, \varepsilon]$, let $\mathcal{R}_{\omega}$ denote the partition into atoms of the form $\alpha-\omega, \alpha \in \mathcal{R}$. Extending by periodicity, we regard $\mathcal{R}, \mathcal{R}_{\omega}$ as partitions on $\mathbb{R}$ as well. Given an interval $J \subset \mathbb{R}$, let us write $\left.\mathcal{R}\right|_{J}=$ $\{\alpha \cap J: \alpha \in \mathcal{R}\}$. For $\omega \in[-\varepsilon, \varepsilon]$, the partition $\left.\mathcal{R}_{\omega}\right|_{J}$ of $J$ is defined analogously.

Lemma 3.4. Assume $\bar{J} \subset \mathbb{R}$ is an interval with $|\bar{J}|<L^{-\beta}$. Let $J$ be the longest atom of $\left.\mathcal{R}\right|_{\bar{J}}$. Then $|J| \geq \kappa|\bar{J}|$, where $\kappa=\min \left\{\frac{1}{5}, K_{1}^{-1}\right\}$.

Proof. Some notation for this proof: given $\hat{x} \in\left\{\tilde{f}^{\prime}=0\right\} \subset \mathbb{R}$ and $0 \leq l \leq k$, define $\mathscr{B}^{l,+}(\hat{x})$ to be the connected component of $\mathscr{B}^{l}$ to the immediate right of $\hat{x}$, and $\mathscr{B}^{l,-}(\hat{x})$ to be the connected component to the immediate left. Let us write $\mathscr{B}(\hat{x})$ for the component of $\mathcal{B}$ containing $\hat{x}$. 
If $\left.\mathcal{R}\right|_{\bar{J}}$ has only one or two atoms of positive length, then $|J| \geq \frac{1}{5}|\bar{J}|$ holds trivially. Hereafter we assume $\left.\mathcal{R}\right|_{\bar{J}}$ consists of three or more atoms of positive length. In particular, $\bar{J}$ contains a connected component of $\mathscr{B}^{l}$ for some $0 \leq l \leq k$, since $|\bar{J}|<L^{-\beta}$ was assumed. Let $\hat{x} \in\left\{\tilde{f}^{\prime}=0\right\}$ be the nearest critical point to $\bar{J}$.

Define

$$
l_{1}=\min \left\{0 \leq l \leq k: \bar{J} \text { contains a component of } \mathscr{B}^{l}\right\} .
$$

There are two cases: (i) $J \subset \mathscr{B}^{l_{1}}$, in which case $J=\mathscr{B}^{l_{1}, \pm}(\hat{x})$ for some choice of \pm , or (ii) $J \cap \mathscr{B}^{l_{1}}=\emptyset$.

For case (i), assume first that $l_{1}=0$. Without loss of generality, we assume $J=$ $\mathscr{B}^{0,+}(\hat{x})$. Note that $\bar{J} \cap \mathcal{E}$ consists of at most two components, hence $|\bar{J} \cap \mathcal{Y}| \leq 2|J|$, while $\bar{J} \cap \mathscr{B}$ has one component, hence $|\bar{J} \cap \mathscr{B}| \leq 2 K_{1}^{-1} L^{-\frac{1}{2}-\beta} \leq 2 L^{-\frac{1}{2}}|J|$. Finally, $\bar{J} \cap \mathcal{I}$ has at most two components, and so $|\bar{J} \cap \mathcal{I}| \leq 2|J|$. In total,

$$
|\bar{J}| \leq|\bar{J} \cap \mathcal{G}|+|\bar{J} \cap \mathcal{I}|+|\bar{J} \cap \mathscr{B}| \leq\left(4+2 L^{-\frac{1}{2}}\right)|J| \leq 5|J| .
$$

Assuming now that $l_{1}>0$, without loss of generality we have $J=\mathscr{B}^{l_{1},+}(\hat{x})$. Moreover, $\bar{J} \subset \bigcup_{i=l_{1}-1}^{k} \mathcal{B}^{l}$; otherwise, $\bar{J}$ would contain an intact component of $\mathcal{B}^{l_{1}-1}$, a contradiction. As before, $\bar{J} \cap \mathscr{B}^{l_{1}-1}$ has at most two components, each of length $\leq|J|$, while $\bar{J} \cap \bigcup_{l_{1}+1}^{k} \mathscr{B}^{l}$ has at most one component of length

$$
\leq 2 K_{1}^{-1} L^{-\frac{l_{1}+1}{2}-\beta} \leq 2 L^{-\frac{1}{2}}|J| \ll|J|,
$$

unless $l_{1}=k$, in which case we can ignore this contribution. As before, we conclude $|\bar{J}| \leq 3|J|$.

For case (ii), if $l_{1}=0$, then $J \subset \mathcal{E}$. Note $\bar{J}$ does contain some atom $\mathscr{B}^{0, \pm}(\hat{x})$, hence $|J| \geq K_{1}^{-1} L^{-\beta}>K_{1}^{-1}|\bar{J}|$, having assumed in Lemma 3.4 that $|\bar{J}|<L^{-\beta}$.

If $l_{1}>0$, then likewise it is not hard to show that $J \subset \mathscr{B}^{l_{1}-1}$. As before, $\bar{J}$ contains some $\mathcal{B}^{l_{1}, \pm}(\hat{x})$ and so $|J| \geq K_{1}^{-1} L^{-\frac{l_{1}}{2}-\beta}$ holds. One now repeats the same arguments as for case (i), $l_{1}>0$.

3.2.2. Proof of Proposition 3.3. To prove Proposition 3.3, we introduce the random interval process $\left(J_{i}\right)_{i \geq 0}$ of subintervals of $\mathbb{R}$, defined as follows. Fix $X_{0} \in S^{1}$. To start, $J_{0}:=X_{0}+[-\varepsilon, \varepsilon]$, regarded as an interval in $\mathbb{R}$. We set $\bar{J}_{1}:=\tilde{f}\left(J_{0}\right)$ and define $J_{1}$ to be the longest atom of $\left.\mathcal{R}_{\omega_{1}}\right|_{J_{1}}$; if more than one atom has maximal length, then select $J_{1}$ to be the rightmost one. Inductively, given $J_{0}, \ldots, J_{i}$, define $\bar{J}_{i+1}:=\tilde{f}_{\omega_{i}}\left(J_{i}\right)$ and $J_{i+1}$ to be the longest atom of $\left.\mathcal{R}_{\omega_{i+1}}\right|_{\bar{J}_{i+1}}$, with the same rule if there is a tie for the longest atom.

We terminate the process $\left(J_{i}\right)_{i}$ at the stopping time $\sigma:=\min \left\{\sigma_{1}, \sigma_{2}\right\}$, where

$$
\sigma_{1}:=\min \left\{i:\left|\bar{J}_{i}\right|>L^{-\beta}\right\}, \quad \sigma_{2}:=\min \left\{i: J_{i} \subset \mathcal{B}_{\omega_{i}}^{k}\right\} .
$$

Lemma 3.5. There exists $\widehat{N}=\widehat{N}(k, \beta) \in \mathbb{N}$ for which $\mathbb{P}_{X_{0}}(\sigma \leq \hat{N}-1)>0$ holds.

Proof of Proposition 3.3 assuming Lemma 3.5. Observe that for each $i \geq 0$,

$$
\bar{J}_{i} \subset \tilde{f}_{\theta \underline{\omega}}^{n-1} \circ \tilde{f}\left(\mathcal{N}_{\varepsilon}\left(X_{0}\right)\right),
$$


hence the projection $\bar{J}_{i}(\bmod 1)$ of $\bar{J}_{i}$ to $S^{1}$ is a subset of the support of the measure $\mathbb{P}_{X_{0}}\left(X_{i} \in \cdot \mid\left\{\omega_{i}\right\}_{i \neq 0}\right)$.

On the event $\sigma=\sigma_{1}=m$ for some $m \geq 0$, it is not hard to see that $\left|\tilde{f}_{\omega_{m}}\left(\bar{J}_{m}\right)\right| \gg 1$ (see Section 2), hence on the event $\left\{\sigma=\sigma_{1}\right\}$ we have $T \leq \sigma_{1}+1$. Meanwhile, $T \leq \sigma_{2}$ holds unconditionally (note $\tilde{X}_{m} \in \mathscr{B}_{\omega_{m}}^{k}$ iff $X_{m} \in \mathcal{B}_{\omega_{m}}^{k}$ ), hence

$$
T \leq \sigma+1
$$

holds almost surely.

To complete the proof of Proposition 3.3, it remains to prove Lemma 3.5.

Proof of Lemma 3.5. We will show that conditioned on $\left\{\sigma_{2}>\widehat{N}\right\}$, we have $\sigma_{1} \leq \hat{N}$.

Define $t_{1}=\min \left\{t: J_{t} \subset \mathscr{B}_{\omega_{t}}\right\}$ and let $p_{1} \in\{1, \ldots, k-1\}$ be such that $J_{t_{1}} \subset \mathscr{B}_{\omega_{t_{1}}}^{p_{1}}$. Inductively, for $j>1$ set

$$
t_{j}=\min \left\{t>t_{j-1}: J_{t} \subset \mathscr{B}_{\omega_{t}}\right\}
$$

and let $p_{j}$ be such that $J_{t_{j}} \subset \mathcal{B}_{\omega_{t_{j}}}^{p_{j}}$. We let $q \geq 0$ be such that $t_{q} \leq \widehat{N}<t_{q+1}$ (note $q=0$ is allowed).

At time $t_{j}$, the interval process $J_{t_{j}}$ is said to initiate a bound period of length $p_{j}$; that is, $J_{t_{j}+1}, \ldots, J_{t_{j}+p_{j}}$ shadow some postcritical orbit in the sense of Lemma 2.1. In particular, $t_{j}+p_{j}+1 \leq t_{j+1}$ for all $j$. For $t_{j}+p_{j}+1 \leq t \leq t_{j+1}$, we say that the interval $J_{t}$ is free.

When $t$ is free, expansion on $\mathcal{E} \cup \mathcal{I}$ (see Section 2) and Lemma 3.4 imply

$$
\left|J_{t+1}\right| \geq \kappa\left|\bar{J}_{t+1}\right| \geq \kappa L^{\frac{1}{2}-\beta}\left|J_{t}\right|
$$

while along bound periods (having conditioned on $\left\{\sigma_{2}>\widehat{N}\right\}$, it follows that $p_{j}<k$ for all $j \leq q$ ) we have

$$
J_{t_{j}+p_{j}+1} \geq \kappa\left|\bar{J}_{t_{j}+p_{j}+1}\right| \geq \kappa L^{\left(\frac{1}{2}-\beta\right)\left(p_{j}+1\right)}\left|J_{t_{j}}\right|
$$

since, by Lemma 2.1, we have $\bar{J}_{t_{j}+p_{j}+1}=\tilde{f}_{\theta^{t_{j}} \underline{\omega}}^{p_{j}+1} J_{t_{j}}$ (i.e., no cutting can occur during a bound period). We obtain that when $J_{t}$ is free, we have

$$
\left|\bar{J}_{t}\right| \geq\left(\kappa L^{\frac{1}{2}-\beta}\right)^{t} \cdot 2 \varepsilon \geq L^{t\left(\frac{1}{2}-2 \beta\right)} \cdot 2 \varepsilon
$$

when $L$ is sufficiently large. Since, for any $t$, the interval $J_{t^{\prime}}$ is free for at least one $t^{\prime} \in$ $\{t, \ldots, t+k\}$, and $\varepsilon \geq L^{-(2 k+1)(1-\beta)+\beta}$ was assumed, it follows that $\sigma_{1} \leq \hat{N}$, where $\widehat{N}=\widehat{N}(k, \beta)$ depends on $k, \beta$ alone.

3.2.3. Proof of Proposition 3.2. Assume $X_{0} \in \mathcal{B}_{\omega_{0}}^{k}$. We form what is essentially the same interval process as before, starting now with the interval

$$
J_{1}:=X_{1}+[-\varepsilon, \varepsilon]
$$


again regarded as a subset of $\mathbb{R}$, and taking $\bar{J}_{2}:=\tilde{f}\left(J_{1}\right)$, and $\left.J_{2} \in \mathcal{R}\right|_{\bar{J}_{2}}$ the longest atom. The intervals $J_{3}, J_{4}, \ldots$ are defined the same as before.

As in the proof of Lemma 3.5, no cutting occurs during the initial bound period of length $k$, hence $\bar{J}_{k+1}=\tilde{f}_{\theta^{2} \underline{\omega}}^{k-1} \circ \tilde{f}\left(\mathcal{N}_{\varepsilon}\left(X_{1}\right)\right)$. By Lemmas 2.1 and 3.4, this implies

$$
\left|J_{k+1}\right| \geq \kappa\left|\bar{J}_{k+1}\right| \geq L^{-(k+1)(1-\beta)+\beta / 2},
$$

perhaps taking $L$ sufficiently large (independently of $k$ ).

With $t_{1}=0, p_{1}=k$ and $t_{j}, p_{j}, j \geq 2$ defined as in the proof of Lemma 3.5, note that if $p_{j}<k$ then (7) holds, while if $t$ is free we have that (6) holds. It remains to check that some interval growth occurs when $p_{j}=k$; we do so below.

Lemma 3.6. Assume $L$ is sufficiently large, depending on $\beta$. Let $J \subset \mathscr{B}_{\omega_{0}}^{k}$ be an interval for which $|J| \geq L^{-(k+1)(1-\beta)+\gamma}$ for some constant $\gamma>\beta / 2$. Then $\left|\tilde{f}_{\underline{\omega}}^{k+1}(J)\right| \geq$ $L^{-(k+1)(1-\beta)+\frac{3}{2} \gamma}$.

Proof. It suffices to estimate the length of $\tilde{f}_{\omega_{0}}(J)$. For this, let us subdivide $J=J^{+} \cup J^{-}$, where $J^{+}$is to the right of the critical point and $J^{-}$to the left. Without loss of generality, let $J^{+}$be the longer of the two intervals, so $\left|J^{+}\right| \geq \frac{1}{2}|J|$ holds.

Writing $J^{+}=\left[\hat{x}-\omega_{0}, \hat{x}-\omega_{0}+b^{+}\right], b^{+}>0\left(\right.$ noting $\left.b^{+} \geq \frac{1}{2}|J|\right)$, we have

$$
(*)=\left|\tilde{f}_{\omega_{0}}\left(J^{+}\right)\right|=\int_{\hat{x}}^{\hat{x}+b^{+}}\left|\tilde{f}^{\prime}(x)\right| d x \geq K_{1} \int_{0}^{b^{+}} x d x=\frac{1}{2} K_{1}\left(b^{+}\right)^{2} \geq \frac{1}{8} K_{1}|J|^{2} .
$$

Plugging in the lower bound for $|J|$ gives

$$
(*) \geq \frac{1}{8} K_{1} L^{-2(k+1)(1-\beta)+2 \gamma} \geq L^{-2(k+1)(1-\beta)+\frac{3}{2} \gamma} .
$$

From here, using Lemma 2.1 we estimate

$$
\left|\tilde{f}_{\underline{\omega}}^{k+1}(J)\right| \geq\left|\tilde{f}_{\underline{\omega}}^{k+1}\left(J^{+}\right)\right| \geq L^{-(k+1)(1-\beta)+\frac{3}{2} \gamma} .
$$

Proposition 3.2 now follows from a similar argument to that for Lemma 3.5, where $N=N(k, \beta) \in \mathbb{N}$ and the constant $c>0$ depends on $N$ as well as $L$. Details are left to the reader.

\section{Itineraries and distortion}

For the remainder of the paper we turn our attention to the proof of Theorem B. In essence, this proof will be an elaboration on the idea, used heavily in Section 3.2, that the predominant expansion of $f=f_{L, a}$ has the effect of amplifying the noise $\varepsilon$. On the other hand, in Section 3.2 and the proof of ergodicity as in Theorem A, we were able to avoid exerting any precise control on the densities of the conditional laws $P^{n}\left(X_{0}, \cdot \mid\left\{\omega_{i}, i \neq 0\right\}\right)$. For 
our purposes in Section 6, however, we will need some control on these densities, which amounts to controlling distortion of the random compositions $f_{\underline{\omega}}^{n}$.

Our objective in Section 4, then, is to establish some control on the distortion of $f_{\underline{\omega}}^{n}$. As is typical of systems exhibiting nonuniform expansion, distortion of $f_{\underline{\omega}}^{n}$ for some $n \geq \overline{1}$ can only be controlled along sufficiently small intervals $J \subset S^{1}$ (see, e.g., [48]). Establishing just how small these intervals need to be is a crucial component of our argument.

In Section 4.1 we formulate itineraries for the random dynamics of $f_{\underline{\omega}}^{n}$, a form of symbolic dynamics for the trajectories of $f_{\underline{\omega}}^{n}$ with the property (checked in Section 4.2) that the distortion of $f_{\underline{\omega}}^{n}$ can be controlled along subintervals with the same itinerary (symbolic sequence) out to time $n-1$.

The preceding paragraphs apply equally well to deterministic as well as random compositions of interval maps - indeed, the assignment of itineraries to control distortion is an old idea (see the references in [48] for more information). Something to keep in mind, however, is that since condition $(\mathrm{H} 3)_{c, k}$ only guarantees bound periods up to length $k$, we lose control of the dynamics of $f_{\underline{\omega}}^{n}$ upon the first visit to the "worst possible" neighborhood $\mathscr{B}^{k}$ of $\left\{f^{\prime}=0\right\}$. Thus the itinerary subdivision procedure and resulting distortion estimates we obtain below are only valid up until this first visit to $\mathscr{B}^{k}$. This issue will be addressed in Section 5.

\subsection{Itineraries}

Throughout, in addition to the preparations in Section 2.1.1, we assume the parameter $\varepsilon$ satisfies the upper bound (3), so that Lemma 2.1 holds. No lower bound on $\varepsilon$ is assumed.

(A) Partition construction. To start, we define the partition $\mathcal{P}$ of $S^{1}$ as follows. Recall the notation $\mathscr{B}^{0}=\mathcal{I}$.

- $\left.\mathcal{P}\right|_{\mathscr{E}}$ is the partition of $\mathcal{E}$ into connected components.

- To define $\left.\mathcal{P}\right|_{B^{l}}, 0 \leq l<k$, start by cutting $\mathscr{B}^{l}$ into connected components. For each such component $J,\left.\mathcal{P}\right|_{J}$ is defined as any partition of $J$ into intervals of length

$$
\in\left[(l+1)^{-2} L^{-\frac{l+3}{2}-\beta}, 2(l+1)^{-2} L^{-\frac{l+3}{2}-\beta}\right] .
$$

- $\left.\mathcal{P}\right|_{\mathcal{B}^{k}}$ is the partition of $\mathscr{B}^{k}$ into connected components.

We write $\mathcal{P}_{\omega}$ for the partition of $S^{1}$ with atoms of the form $C-\omega, C \in \mathcal{P}$. Abusing notation somewhat, we regard $\mathcal{P}, \mathcal{P}_{\omega}$ as partitions of $\mathbb{R}$, extended by periodicity.

Definition 4.1. For a bounded, connected interval $I \subset S^{1}$ (or $\subset \mathbb{R}$ ) which is not a singleton, we define the partition $\mathcal{P}_{\omega}(I)$ of $I$ as follows. To start, form $\left.\mathcal{P}_{\omega}\right|_{I}=\left\{J \cap I: J \in \mathcal{P}_{\omega}\right.$, $J \cap I \neq \emptyset\}$, and write $J_{1}, J_{1}, \ldots, J_{N}$ for the nonsingleton atoms of this partition in increasing order from left to right (note that $N=1$ is possible).

- If $N=1,2$ or 3 , then set $\mathcal{P}_{\omega}(I):=\{I\}$.

- If $N \geq 4$, then set $\mathcal{P}_{\omega}(I)=\left\{J_{1} \cup J_{2}, J_{3}, J_{4}, \ldots, J_{N-2}, J_{N-1} \cup J_{N}\right\}$. 
We define the bound period $p(I)$ of an interval $I$ as follows. First, $p: S^{1} \rightarrow\{0, \ldots, k\}$ (or $\mathbb{R} \rightarrow\{0, \ldots, k\}$ ) is defined by setting $\left.p\right|_{\mathscr{B}^{p}}:=p$ for all $1 \leq p \leq k$, and $\left.p\right|_{I} \cup \mathcal{E}=0$. Next, for an interval $I \subset S^{1}$ or $\mathbb{R}$, we define

$$
p(I)=\max _{x \in I} p(x) .
$$

For $\omega \in[-\varepsilon, \varepsilon]$, we define $p_{\omega}(\cdot)=p(\cdot-\omega)$.

Remark 4.2. For an atom $C \in \mathcal{P}$ or $\mathcal{P}_{\omega}$, write $C^{+}$for the union of $C$ with its two adjacent atoms. Observe that for any interval $I$, we have that each atom $J \in \mathcal{P}_{\omega}(I)$ is contained in $C^{+}$for some $C \in \mathcal{P}_{\omega}(I)$. By this line of reasoning, for any $J \in \mathcal{P}_{\omega}(I)$ with $p=p(J) \in\{1, \ldots, k-1\}$, we have the estimate

$$
|J| \leq 6 p^{-2} L^{-\frac{p+2}{2}-\beta} .
$$

Similarly, if $J \in \mathcal{P}_{\omega}(I), J \cap \mathcal{B}_{\omega}^{k} \neq \varnothing$ (i.e., $p(J)=k$ ) then $|J| \leq 3 \max \left\{1, K_{1}^{-1}\right\} L^{-\frac{k}{2}-\beta}$.

For a lower bound, if in the above setting we have that there are at least two distinct atoms in $\mathcal{P}_{\omega}(I)$, then any atom $J \in \mathcal{P}_{\omega}(I)$ with $p=p_{\omega}(J)>0$ must contain an atom $\left.C \in \mathcal{P}_{\omega}\right|_{\mathcal{B}^{p}}$. Thus

$$
|J| \geq(p+1)^{-2} L^{-\frac{p+3}{2}-\beta} .
$$

Remark 4.3. Fix a sample $\underline{\omega} \in \Omega$ and let $J$ be a connected interval contained in $C^{+}$for some $C \in \mathcal{P}_{\omega_{0}}$. If $p:=p_{\omega_{0}}(J)>0$, then

$$
\tilde{f}_{\underline{\omega}}^{i}(J) \subset \mathcal{G} \text { for all } 1 \leq i \leq p,
$$

even though $J$ is not necessarily a subset of $\mathcal{B}_{\omega_{0}}^{p}$. This is because $\left.\mathcal{P}\right|_{\mathcal{B}^{p-1} \text {-atoms are small }}$ enough so that $J \subset B\left(-\frac{p+\beta}{2}\right)$ must hold, Lemma 2.1 implies that $f_{\underline{\omega}}^{i}\left(B\left(-\frac{p+\beta}{2}\right)\right) \subset \mathcal{E}$ for all $1 \leq i \leq p$ and all samples $\underline{\omega}$. Note, in particular, that $\tilde{f}_{\underline{\omega}}^{i}(J)$ meets at most one component of $\mathcal{G}$ for each $1 \leq i \leq p$, hence $\mathcal{P}_{\omega_{i}}\left(\tilde{f}_{\underline{\omega}}^{i}(J)\right)=\left\{\tilde{f}_{\underline{\omega}}^{i}(J)\right\}$.

(B) Time-n itineraries for an interval $I \subset S^{\mathbf{1}}$. Let $I \subset S^{1}$ be an interval (which we regard as a subset of $\mathbb{R}$ ) and fix a sample $\underline{\omega} \in \Omega$. For each time $i \geq 1$, we define a partition $\mathcal{Q}_{i}=\mathcal{Q}_{i}\left(I ;\left(\omega_{0}, \ldots, \omega_{i}\right)\right)$ of $I$, the atoms of which correspond to points in $I$ with the same itinerary for the map $\tilde{f}_{\underline{\omega}}^{i+1}$.

The definition is inductive. To start, we define $\mathcal{Q}_{0}=\mathcal{P}_{\omega_{0}}(I)$. Assuming $\mathcal{Q}_{0}, \mathcal{Q}_{1}, \ldots, \mathcal{Q}_{i}$ have been constructed, for each $C_{i} \in \mathcal{Q}_{i}$ we define $\mathcal{Q}_{i+1} \geq \mathcal{Q}_{i}$ as follows: ${ }^{2}$

$$
\left.\mathcal{Q}_{i+1}\right|_{C}=\left(\tilde{f}_{\underline{\omega}}^{i+1}\right)^{-1}\left(\mathcal{P}_{\omega_{i+1}}\left(\tilde{f}_{\underline{\omega}}^{i+1}\left(C_{i}\right)\right)\right) .
$$

In what follows, we will only attempt to keep track of itineraries until a first "near visit" to the set $\mathscr{B}^{k}$. Precisely, we define a "terminating" stopping time $\tau=\tau[I]: I \times \Omega \rightarrow$ $\mathbb{Z}_{\geq 0} \cup\{\infty\}$ as

$$
\tau(x, \underline{\omega})=\min \left\{i \geq 0: f_{\underline{\omega}}^{i}\left(C_{i}(x)\right) \cap \mathscr{B}_{\omega_{i}}^{k} \neq \emptyset\right\} .
$$

\footnotetext{
${ }^{2}$ Here, for two partitions $\zeta$, $\xi$, we write $\zeta \leq \xi$ if each atom of $\zeta$ is a union of $\xi$-atoms.
} 
Here, $C_{i}(x)$ denotes the $\mathcal{Q}_{i}$-atom containing $x$. Notice that $\tau$ is adapted to $\left(\mathcal{Q}_{i}\right)_{i}$, i.e., $\{\tau>i\}$ is a union of $Q_{i}$-atoms for each $i \geq 0$. In particular, $\{\tau>i\}$ depends only on $\omega_{0}, \ldots, \omega_{i}$.

(C) Bound and free periods of an itinerary. Fix $n \geq 1$ and $C_{n} \in \mathcal{Q}_{n}$ such that $\left.\tau\right|_{C_{n}} \geq n$. For each $i<n$, let $C_{i} \in Q_{i}$ denote the atom containing $C_{n}$. For $1 \leq i \leq n$, we write $I_{i}=\tilde{f}_{\underline{\omega}}^{i}\left(C_{i}\right)$.

Define

$$
\begin{aligned}
& t_{1}=\min \{n\} \cup\left\{i \geq 0: I_{i} \cap \mathcal{B}_{\omega_{i}} \neq \emptyset\right\}, \\
& t_{j}=\min \{n\} \cup\left\{i>t_{j-1}: I_{i} \cap \mathcal{B}_{\omega_{i}} \neq \emptyset\right\} \quad \text { for } j \geq 2,
\end{aligned}
$$

and let $q \geq 0$ be the index for which $t_{q+1}=n$. For $1 \leq j \leq q$, define

$$
p_{j}=p_{\omega_{t_{j}}}\left(I_{t_{j}}\right)
$$

At time $t_{j}, 1 \leq j \leq q$, the itinerary $C_{n}$ initiates a bound period of length $p_{j}$ (Remark 4.3); in particular, $t_{j}+p_{j}<t_{j+1}$ for all $1 \leq j<q$. We say that $C_{n}$ is bound at time $t$ if $t \in\left[t_{j}+1, t_{j}+p_{j}\right]$ for some $1 \leq j<q$ and that $C_{n}$ is free at time $t$ if it is not bound at time $t$.

By Remark 4.3 and the fact that $\left.\tau\right|_{C_{n}} \geq n$, we have the following.

Lemma 4.4. Let $1 \leq i \leq n$ and assume $C_{n} \in \mathcal{Q}_{n}$ is such that $\left.\tau\right|_{C_{n}} \geq n$.

(a) If $C_{n}$ is free at time $i$, then

$$
\left|\left(f_{\underline{\omega}}^{i}\right)^{\prime}\right| C_{n} \mid \geq L^{i\left(\frac{1}{2}-\beta\right)} .
$$

(b) If $C_{n}$ is bound at time $i$, i.e., $i \in\left[t_{j}+1, t_{j}+p_{j}\right]$ for some $1 \leq j \leq q$, then

$$
\left|\left(f_{\underline{\omega}}^{i}\right)^{\prime}\right| C_{n} \mid \geq L^{t_{j}\left(\frac{1}{2}-\beta\right)+(1-\beta)\left(i-\left(t_{j}+1\right)\right)-\frac{p_{j}-1}{2}-\beta} .
$$

In this case, $C_{t_{j}}=C_{t_{j}+1}=\cdots=C_{t_{j}+p_{j}}=C_{i}$ and $C_{n}$ is free at time $t_{j}+p_{j}+1$. Note that $C_{t_{j}+p_{j}+1} \subsetneq C_{i}$ is possible.

\subsection{Distortion estimates}

Let $I \subset S^{1}$ be a connected interval, $\underline{\omega} \in \Omega$ a sample. Assume that the partitions $\left(\mathcal{Q}_{i}\right)_{i \geq 0}$, $\mathcal{Q}_{i}=\mathcal{Q}_{i}\left(I ;\left(\omega_{0}, \ldots, \omega_{i}\right)\right)$ and the stopping time $\tau=\tau[I]$ have been constructed as in Section 4.1. Here we prove a time- $n$ distortion estimate for trajectories with the same time- $n$ itineraries, i.e., belonging to the same $\mathcal{Q}_{n}$-atom.

Our approach to distortion estimates is inspired from the treatment in [48], which in turn is a version of estimates first appearing in $[4,5]$.

Proposition 4.5. For all $L$ sufficiently large, the following holds. Let $n \geq 1$. Assume $C_{n} \in$ $Q_{n}$ is free at time $n$ and $\left.\tau\right|_{C_{n}} \geq n$. Let $x, x^{\prime} \in C_{n}$. Then

$$
\frac{\left(\tilde{f}_{\underline{\omega}}^{n}\right)^{\prime}(x)}{\left(\tilde{f}_{\underline{\omega}}^{n}\right)^{\prime}\left(x^{\prime}\right)} \leq e^{K_{2} L^{-\frac{1}{2}}+4\left\|\psi^{\prime \prime}\right\|_{C_{0}} L^{2 \beta}\left|\tilde{f}_{\underline{\omega}}^{n} x-\tilde{f}_{\underline{\omega}}^{n} x^{\prime}\right|} .
$$


We start with a preliminary lemma.

Lemma 4.6. Let $L$ be sufficiently large, and let $\eta \in\left[-\frac{3}{4}, 0\right]$. Let $y, y^{\prime} \in S^{1}, i \geq 1$, and define $J$ to be the interval between $y, y^{\prime}$. If $f_{\underline{\omega}}^{j}(J) \subset B(\eta)^{c}$ for all $0 \leq j<i$, then

$$
\left|\log \frac{\left(f_{\underline{\omega}}^{i}\right)^{\prime}(y)}{\left(f_{\underline{\omega}}^{i}\right)^{\prime}\left(y^{\prime}\right)}\right| \leq 2\left\|\psi^{\prime \prime}\right\|_{C^{0}} L^{-1-2 \eta}\left|f_{\underline{\omega}}^{i}(y)-\tilde{f}_{\underline{\omega}}^{i}\left(y^{\prime}\right)\right| .
$$

Proof. Define $y_{j}=\tilde{f}_{\underline{\omega}}^{j} y, y_{j}^{\prime}=\tilde{f}_{\underline{\omega}}^{j} y^{\prime}$. We estimate

$$
\begin{aligned}
(*):=\left|\log \frac{\left(f_{\underline{\omega}}^{i}\right)^{\prime}(y)}{\left(f_{\underline{\omega}}^{i}\right)^{\prime}\left(y^{\prime}\right)}\right| & \leq \sum_{j=0}^{i-1}\left|\log \frac{\left(f_{\omega_{j+1}}\right)^{\prime}\left(y_{j}\right)}{\left(f_{\omega_{j+1}}\right)^{\prime}\left(y_{j}^{\prime}\right)}\right| \\
& \leq \sum_{j=0}^{i-1} \frac{L\left\|\psi^{\prime \prime}\right\|_{C_{0}}}{L^{1+\eta}}\left|y_{j}-y_{j}^{\prime}\right|=\left\|\psi^{\prime \prime}\right\|_{C^{0}} L^{-\eta} \sum_{j=0}^{i-1}\left|y_{j}-y_{j}^{\prime}\right| .
\end{aligned}
$$

We bound $\left|y_{j}-y_{j}^{\prime}\right| \leq L^{-(1+\eta)(i-j)}\left|y_{i}-y_{i}^{\prime}\right|$, hence

$$
(*) \leq\left\|\psi^{\prime \prime}\right\|_{C^{0}} L^{-\eta}\left(\sum_{j=0}^{i-1} L^{-(1+\eta)(i-j)}\right)\left|y_{i}-y_{i}^{\prime}\right| \leq 2\left\|\psi^{\prime \prime}\right\|_{C^{0}} L^{-1-2 \eta}\left|y_{i}-y_{i}^{\prime}\right| .
$$

In view of (1), observe that the above estimates can be written in the following alternative form: writing $J_{j}$ for the interval between $y_{j}, y_{j}^{\prime}$, we have

$$
\sum_{j=0}^{i-1} \frac{\left|J_{j}\right|}{d\left(J_{j}, C_{\psi}^{\prime}-\omega_{j}\right)} \leq 2\left\|\psi^{\prime \prime}\right\|_{C^{0}} L^{-1-2 \eta}\left|J_{i}\right|
$$

Proof of Proposition 4.5. Below, we write $C$ to refer to a generic positive constant; the value of $C$ may change from line to line, but always depends only on the function $\psi$.

With $n \geq 1$ and $C_{n} \in \mathcal{Q}_{n}$ fixed and free at time $n$, we adopt the notation of Section $4.1(\mathrm{C})$. Write $x_{i}=\tilde{f}_{\underline{\omega}}^{i}(x), x_{i}^{\prime}=\tilde{f}_{\underline{\omega}}^{i}\left(x^{\prime}\right)$. By hypothesis, $x, x^{\prime}$ belong to the same $\mathcal{Q}_{i}$ atom $C_{i}$ for all $0 \leq i \leq n$.

We decompose

$$
\left|\log \frac{\left(\tilde{f}_{\underline{\omega}}^{n}\right)^{\prime}(x)}{\left(\tilde{f}_{\underline{\omega}}^{n}\right)^{\prime}\left(x^{\prime}\right)}\right| \leq \sum_{i=0}^{n-1}\left|\log \frac{\tilde{f}_{\omega_{i}^{\prime}}\left(x_{i}\right)}{\tilde{f}_{\omega_{i}^{\prime}}\left(x_{i}^{\prime}\right)}\right| .
$$

Using (1), each summand is bounded by

$$
\left|\log \frac{\tilde{f}_{\omega_{i}}^{\prime}\left(x_{i}\right)}{\tilde{f}_{\omega_{i}}^{\prime}\left(x_{i}^{\prime}\right)}\right| \leq C \frac{\left|J_{i}\right|}{d\left(J_{i}, C_{\psi}^{\prime}-\omega_{i}\right)}
$$

where $J_{i}$ is the interval from $x_{i}+\omega_{i}$ to $x_{i}^{\prime}+\omega_{i}$. 
With $t_{j}, p_{j}$ as in (8), (9), we decompose the time interval from 0 to $n$ into the succession of free and bound periods experienced by the atom $C_{n} \in \mathcal{Q}_{n}$ containing $x, x^{\prime}$ :

$$
0 \leq t_{1}<t_{1}+p_{1}<t_{2}<t_{2}+p_{2}<\cdots<t_{q}<t_{q}+p_{q}<t_{q+1}:=n .
$$

We assume going forward that $q \geq 1$, i.e., $C_{n}$ experiences at least one bound period. If $q=0$, then Proposition 4.5 follows easily from Lemma 4.6 applied to $\eta=-\frac{1}{2}-\beta$; details are left to the reader.

We now decompose $\sum_{i=0}^{n-1}$ as follows:

$$
\sum_{i=0}^{n-1} \frac{\left|J_{i}\right|}{d\left(J_{i}, C_{\psi}^{\prime}-\omega_{i}\right)}=\sum_{i=0}^{t_{1}-1}+\sum_{j=1}^{q}\left(\sum_{i=t_{j}}^{t_{j}+p_{j}}+\sum_{i=t_{j}+p_{j}+1}^{t_{j+1}-1}\right)=: D_{0}^{\prime}+\sum_{j=1}^{q}\left(D_{j}+D_{j}^{\prime}\right) .
$$

Above, a summand of the form $\sum_{m}^{m-1}, m \in \mathbb{N}$ is regarded as empty and the corresponding summation is defined to be 0 (as may happen for some of the $D_{j}^{\prime}$ terms). The $D_{j}, D_{j}^{\prime}$ are estimated separately below.

Before proceeding, observe now that $\left|J_{t_{j}+p_{j}+1}\right| \geq L^{\left(p_{j}+1\right)\left(\frac{1}{2}-\beta\right)}\left|J_{t_{j}}\right|$ and $\left|J_{t+1}\right| \geq$ $L^{\frac{1}{2}-\beta}\left|J_{t}\right|$ for all $t$ such that $C_{t}, C_{t+1}$ are free. In particular,

$$
\left|J_{t_{j+1}}\right| \geq L^{\left(t_{j+1}-t_{j}\right)\left(\frac{1}{2}-\beta\right)}\left|J_{t_{i}}\right|
$$

for all $1 \leq i \leq q$.

Bounding $\sum_{j=1}^{q} D_{j}$. Let $1 \leq j \leq q$.

Claim 4.7. We claim that

$$
\sum_{i=t_{j}+1}^{t_{j}+p_{j}} \frac{\left|J_{i}\right|}{\operatorname{dist}\left(J_{i}, C_{\psi}^{\prime}-\omega_{i}\right)} \leq C L^{2 \beta} \frac{\left|J_{t_{j}}\right|}{d\left(J_{t_{j}}, C_{\psi}^{\prime}-\omega_{t_{j}}\right)}
$$

Assuming the claim, we now bound $\sum_{j=1}^{q} D_{j}$. For $1 \leq p<k$, let $\mathcal{K}_{p}=\{1 \leq j \leq q$ : $\left.p_{j}=p\right\}$. Let $j_{p}^{*}=\max \mathcal{K}_{p}$, and observe that $\left|J_{t_{j}}\right| \leq\left|J_{t_{j_{p}^{*}}}\right| \cdot L^{-\left(t_{j_{p}^{*}}-t_{j}\right)\left(\frac{1}{2}-\beta\right)}$ for all $j \in \mathcal{K}_{p}$ by (10). Thus,

$$
\begin{aligned}
\sum_{j \in \mathcal{K}_{p}} D_{j} \leq C L^{2 \beta} \sum_{j \in \mathcal{K}_{p}} \frac{\left|J_{t_{j}}\right|}{\operatorname{dist}\left(J_{t_{j}}, C_{\psi}^{\prime}-\omega_{t_{j}}\right)} & \leq \frac{C L^{2 \beta}}{1-L^{-\left(\frac{1}{2}-\beta\right)}} \cdot \frac{\left|J_{t_{j_{p}^{*}}}\right|}{\frac{1}{2} K_{1}^{-1} L^{-\frac{p+1}{2}-\beta}} \\
& \leq C L^{2 \beta} \frac{\left|J_{t_{j_{p}^{*}}}\right|}{L^{-\frac{p+1}{2}-\beta}} .
\end{aligned}
$$

Here we are using that $\operatorname{dist}\left(J_{t_{j}}, C_{\psi}^{\prime}-\omega_{t_{j}}\right) \geq \frac{1}{2} K_{1}^{-1} L^{-\frac{p+1}{2}-\beta}$ for all $j \in \mathcal{K}_{p}$. By Remark 4.2, we have $\left|J_{t_{j_{p}^{*}}}\right| \leq 6 p^{-2} L^{-\frac{p+2}{2}-\beta}$. So,

$$
\sum_{j \in \mathcal{K}_{p}} D_{j} \leq C L^{2 \beta} \frac{p^{-2} L^{-\frac{p+2}{2}-\beta}}{L^{-\frac{p+1}{2}-\beta}} \leq C p^{-2} L^{-\frac{1}{2}+2 \beta},
$$


hence

$$
\sum_{j=1}^{q} D_{j}=\sum_{p=1}^{k-1} \sum_{j \in \mathcal{K}_{p}} D_{j} \leq \sum_{p=1}^{k-1} C p^{-2} L^{-\frac{1}{2}+2 \beta} \leq C L^{-\frac{1}{2}+2 \beta}
$$

Proof of Claim. Assume $I_{t_{j}}$ meets the component of $\mathcal{B}_{\omega_{t_{j}}}$ near $\hat{x}_{t_{j}} \in C_{\psi}^{\prime}-\omega_{t_{j}}$; write $\hat{x}_{i}=\tilde{f}_{\theta^{t_{j}} \underline{\omega}}^{i-t_{j}}\left(\hat{x}_{t_{j}}\right)$ for $i>t_{j}$. Assume, without loss, that

$$
\left|x_{t_{j}}^{\prime}-\hat{x}_{t_{j}}\right| \leq\left|x_{t_{j}}-\hat{x}_{t_{j}}\right|
$$

in the alternative case, exchange the roles of $x_{i}, x_{i}^{\prime}$ in what follows.

For $t_{j}<i \leq t_{j}+p_{j}$, we have

$$
\frac{\left|J_{i}\right|}{\operatorname{dist}\left(J_{i}, C_{\psi}^{\prime}-\omega_{i}\right)}=\frac{\left|x_{i}-x_{i}^{\prime}\right|}{\left|x_{i}-\hat{x}_{i}\right|} \cdot \frac{\left|x_{i}-\hat{x}_{i}\right|}{\operatorname{dist}\left(J_{i}, C_{\psi}^{\prime}-\omega_{i}\right)} .
$$

By Lemmas 2.1 and 4.6, we have that the first right-hand factor is

$$
\leq 2 \frac{\left|x_{t_{j}+1}-x_{t_{j}+1}^{\prime}\right|}{\left|x_{t_{j}+1}-\hat{x}_{t_{j}+1}\right|}
$$

The numerator of (12) coincides with $\left|f_{\omega_{t_{j}}}^{\prime}(\zeta)\right| \cdot\left|x_{t_{j}}-x_{t_{j}}^{\prime}\right|$ for some $\zeta \in J_{t_{j}}$. Moreover,

$$
\left|f_{\omega_{t_{j}}}^{\prime}(\zeta)\right|=\left|f_{\omega_{t_{j}}}^{\prime \prime}\left(\zeta^{\prime}\right)\right| \cdot\left|\zeta-\hat{x}_{t_{j}}\right| \leq L\left\|\psi^{\prime \prime}\right\|_{C^{0}}\left|\zeta-\hat{x}_{t_{j}}\right|
$$

for some $\zeta^{\prime}$ between $\zeta$ and $\hat{x}_{t_{j}}$. By (11) we have $\left|\zeta-\hat{x}_{t_{j}}\right| \leq\left|x_{t_{j}}-\hat{x}_{t_{j}}\right|$, and so conclude that the numerator of (12) is $\leq L\left\|\psi^{\prime \prime}\right\|_{C^{0}} \cdot\left|x_{t_{j}}-\hat{x}_{t_{j}}\right| \cdot\left|J_{t_{j}}\right|$.

For the denominator of (12), we have

$$
\left|x_{t_{j}+1}-\hat{x}_{t_{j}+1}\right|=\frac{1}{2}\left|f_{\omega_{t_{j}}}^{\prime \prime}\left(\zeta^{\prime \prime}\right)\right| \cdot\left|x_{t_{j}}-\hat{x}\right|^{2}
$$

for some $\zeta^{\prime \prime}$ between $x_{t_{j}}$ and $\hat{x}$. For $L$ sufficiently large and all $\varepsilon$ satisfying (3), we have that $\min _{z \in \mathcal{N}_{\varepsilon}(\mathscr{B})}\left|\psi^{\prime \prime}(z)\right| \geq \frac{1}{2} \min \left\{\left|\psi^{\prime \prime}(\hat{z})\right|: \hat{z} \in C_{\psi}^{\prime}\right\}=: c_{1}$ from (H1), (H2). We have therefore that the denominator of (12) is $\geq \frac{1}{2} c_{1} L\left|x_{t_{j}}-\hat{x}_{t_{j}}\right|^{2}$.

Collecting,

$$
\frac{\left|J_{i}\right|}{\operatorname{dist}\left(J_{i}, C_{\psi}^{\prime}-\omega_{i}\right)} \leq C \frac{\left|J_{t_{j}}\right|}{\operatorname{dist}\left(J_{t_{j}}, C_{\psi}^{\prime}-\omega_{t_{j}}\right)} \cdot \frac{\left|x_{i}-\hat{x}_{i}\right|}{\operatorname{dist}\left(J_{i}, C_{\psi}^{\prime}-\omega_{i}\right)},
$$

since $\left|x_{t_{j}}-\hat{x}_{t_{j}}\right|^{-1} \leq d\left(J_{t_{j}}, C_{\psi}^{\prime}-\omega_{t_{j}}\right)^{-1}$ by assumption, and so

$$
\sum_{i=t_{j}+1}^{t_{j}+p_{j}} \frac{\left|J_{i}\right|}{d\left(J_{i}, C_{\psi}^{\prime}-\omega_{i}\right)} \leq C \frac{\left|J_{t_{j}}\right|}{\operatorname{dist}\left(J_{t_{j}}, C_{\psi}^{\prime}-\omega_{t_{j}}\right)}\left(\sum_{i=t_{j}+1}^{t_{j}+p_{j}} \frac{\left|x_{i}-\hat{x}_{i}\right|}{\operatorname{dist}\left(J_{i}, C_{\psi}^{\prime}-\omega_{i}\right)}\right) .
$$

By Lemma 4.6 applied to $\eta=-\beta$, the parenthetical sum is bounded $\leq C L^{-1+2 \beta} \times$ $\left|x_{t_{j}+p_{j}+1}-\hat{x}_{t_{j}+p_{j}+1}\right|$. Since $\left|x_{t_{j}+p_{j}}-\hat{x}_{t_{j}+p_{j}}\right| \leq L^{-\beta / 2} \ll 1$ (see the proof of Lemma 2.1), we bound $\left|x_{t_{j}+p_{j}+1}-\hat{x}_{t_{j}+p_{j}+1}\right| \leq C L$, hence the parenthetical sum is $\leq C L^{2 \beta}$. This completes the proof. 
Bounding $\sum_{j=0}^{q} D_{j}^{\prime}$. For each $1 \leq j<q$, we have from Lemma 4.6 applied to $\eta=$ $-\frac{1}{2}-\beta$ that

$$
D_{j}^{\prime} \leq L^{1-2\left(-\frac{1}{2}-\beta\right)}\left|J_{t_{j+1}}\right|=C L^{2 \beta}\left|J_{t_{j+1}}\right| .
$$

Similarly, we estimate $D_{0}^{\prime} \leq C L^{2 \beta}\left|J_{t_{1}}\right|$. Since $\left|J_{t_{j}}\right| \leq L^{-\left(n-t_{j}\right)\left(\frac{1}{2}-\beta\right)}\left|J_{n}\right|$ for all $1 \leq$ $j \leq q$ by (10), we conclude $\sum_{i=0}^{q} D_{j}^{\prime} \leq C L^{2 \beta}\left|J_{n}\right|$. The proof of Proposition 4.5 is now complete.

\section{Selective averaging process}

We aim to get more refined control on the conditional laws $P^{n}\left(X_{0}, \cdot \mid\left\{\omega_{i}, i \neq 0\right\}\right), n \geq 0$. Towards this end, the itinerary subdivision procedure in Section 4 applied to $I=X_{0}+$ $[-\varepsilon, \varepsilon]$ can be used to control the density of $P^{n}\left(X_{0}, \cdot \mid\left\{\omega_{i}, i \neq 0\right\}, X_{0}+\omega_{0} \in C_{n}\right)$ for some $C_{n} \in \mathcal{Q}_{n}$, i.e., conditioning on $X_{0}+\omega_{0}$ belonging to a single subdivision $C_{n}$ of $\mathcal{Q}_{n}$. This is only valid, however, up until the first "near visit" to $\mathscr{B}^{k}$, the closest neighborhood to the critical set $\left\{f^{\prime}=0\right\}$. Afterwards, the material in Section 4 is no longer valid and we lose control over distortion, hence over the conditioned law $P^{n}\left(X_{0}, \cdot \mid\left\{\omega_{i}, i \neq 0\right\}\right)$.

A rough idea of how to proceed is as follows: visits to $\mathscr{B}^{k}$ "spoil" the random parameter $\omega_{0}$, and so if $X_{m}$ comes too close to $\mathscr{B}^{k}$ for some $m \geq 0$, we will "freeze" $\omega_{0}$ (essentially, treat as deterministic) and "smear" (average) in the perturbation $\omega_{m+1}$, i.e., for $n \geq m$, work with the conditional law $P^{n}\left(X_{0}, \cdot \mid\left\{\omega_{i}, i \neq m+1\right\}\right)$.

Let us make all this more precise. Fix $X_{0} \in S^{1}$ and define the Markov chain $\left(\tilde{X}_{n}\right)$ on $\mathbb{R}$ by $\tilde{X}_{n}=\tilde{f}_{\omega}^{n}\left(X_{0}\right)=\tilde{f}_{\omega_{n-1}}\left(\tilde{X}_{n-1}\right)$. We will obtain in this section an increasing filtration $\left(\mathscr{H}_{n}\right)_{n \geq 0}, \mathscr{H}_{n} \subset \mathscr{F}_{n}:=\sigma\left(\omega_{0}, \omega_{1}, \ldots, \omega_{n}\right)$ (depending also on $\left.X_{0}\right)$, designed so that the conditional measures

$$
v_{n}(\cdot):=\mathbb{P}\left(\tilde{X}_{n} \in \cdot \mid \mathscr{H}_{n}\right)
$$

have the following desirable properties:

(i) the measures $v_{n}$ are absolutely continuous;

(ii) $\quad \rho_{n}:=\frac{d v_{n}}{d \text { Leb }}$ is more or less constant on the interval of support $I_{n}:=\operatorname{supp} v_{n}$;

(iii) the intervals $I_{n}=\operatorname{supp}\left(v_{n}\right)$ are, for large $n$, rather long with high probability.

In this section we focus on the construction of $\mathscr{H}_{n}, I_{n}, v_{n}$ as above; property (ii) will fall out as a natural consequence of our construction and the distortion estimate in Proposition 4.5.

The plan is as follows: first, in Section 5.1 we will describe an algorithm constructing the supporting intervals $I_{n}$ as above, in a way completely parallel to the itinerary construction given in Section 4.1. From this construction, it will be clear when "smearing" in a new $\omega_{i}$ is necessary: this decision is made according to a sequence $\tau_{1}<\tau_{2}<\cdots$ of stopping times roughly related to the first arrival to the neighborhood $\mathscr{B}^{k}$ (closely related to the stopping time $\tau$ as in Section 4.1). In Section 5.2 we will construct the filtration $\left(\mathscr{H}_{n}\right)$ and then describe the resulting conditional measures $v_{n}$ in Section 5.3. 
In addition to the preparations in Section 2.1.1, we assume the parameter $\varepsilon$ satisfies (3), so that Lemma 2.1 holds. No lower bound on $\varepsilon$ is assumed.

\subsection{The supporting intervals $I_{n}$}

We define here an interval ${ }^{3}$-valued stochastic process $\left(I_{n}\right)_{n \geq 1}$ for which $I_{n} \subset \mathbb{R}$ is $\mathcal{F}_{n}$ measurable for all $n$.

Embed $X_{0}=: \tilde{X}_{0} \in \mathbb{R}$ via the identification $S^{1} \cong[0,1)$. Throughout, the dependence of the $I_{n}$ on the sample $\underline{\omega}=\left(\omega_{i}\right)_{i \geq 0} \in \Omega$ is implicit (keeping in mind that $I_{n}$ depends on $\left.\omega_{i}, 0 \leq i \leq n\right)$.

Base cases. We set $I_{0}=\tilde{X}_{0}+[-\varepsilon, \varepsilon]$. To determine $I_{1}$, there are two cases:

- If $I_{0} \cap \mathscr{B}_{\omega_{0}}^{k} \neq \emptyset$, then define $I_{1}=\tilde{X}_{1}+[-\varepsilon, \varepsilon]$.

- Otherwise, form $\mathcal{P}_{\omega_{1}}\left(\tilde{f}\left(I_{0}\right)\right)$ and let $I_{1}$ be the atom containing $\tilde{X}_{1}$.

Note that since $\varepsilon>0$ is assumed to satisfy (3), we have automatically that $\mathcal{P}\left(I_{0}\right)$ consists of a single atom.

Inductive step . Assume the intervals $I_{0}, I_{1}, \ldots, I_{n}$ have been constructed, with $n \geq 1$.

(a) If $I_{n} \cap \mathscr{B}_{\omega_{n}}^{k}=\emptyset, I_{n-1} \cap \mathcal{B}_{\omega_{n-1}}^{k}=\emptyset$, then form $\mathcal{P}_{\omega_{n+1}}\left(\tilde{f}_{\omega_{n}}\left(I_{n}\right)\right)$ and define $I_{n+1}$ to be the atom containing $\widetilde{X}_{n+1}$.

(b) If $I_{n} \cap \mathcal{B}_{\omega_{n}}^{k} \neq \emptyset$, then define $I_{n+1}=\tilde{X}_{n+1}+[-\varepsilon, \varepsilon]$. Form $\mathcal{P}_{\omega_{n+2}}\left(\tilde{f}\left(I_{n+1}\right)\right)$ and let $I_{n+2}$ be the atom containing $\tilde{X}_{n+2}$.

From Lemma 2.1 and Remark 4.3, it is simple to check that cases (a)-(b) are exhaustive and mutually exclusive. Note in case (b) that $I_{n+1} \subset \mathcal{E}_{\omega_{n+1}}$ holds (Lemma 2.1 and (3)).

Definition 5.1. We define a sequence of $\left(\mathcal{F}_{n}\right)$-adapted stopping times $0=$ : $\tau_{0}<\tau_{1}<\tau_{2}<$ $\cdots$ as follows: for $i>0$, set

$$
\tau_{i}=\min \left\{m>\tau_{i-1}: I_{m} \cap \mathcal{B}_{\omega_{m}}^{k} \neq \emptyset\right\} .
$$

Observe that case (b) above is observed iff $n=\tau_{i}$ for some $i$.

As formulated below, between "near visits" to $\mathscr{B}^{k}$ (i.e., the times $\tau_{1}, \tau_{2}, \ldots$ ), the procedure defining the $\left(I_{n}\right)$ process is completely parallel to the itinerary construction in Section 4.1. The proof is straightforward and left to the reader.

Lemma 5.2. Fix $i \geq 0$ and $0 \leq m<n$.

(a) On the event $S_{i, m, n}=\left\{\tau_{i}=m, \tau_{i+1} \geq n\right\}$, we have that the random interval $I_{n}$ is given as

$$
I_{n}=\tilde{f}_{\theta^{m+2} \underline{\omega}}^{n-m-2} \circ \tilde{f}(\widehat{C}),
$$

where $\widehat{C}$ is the atom of $\mathcal{Q}_{n-m-1}\left(I_{m+1} ;\left(0, \omega_{m+2}, \ldots, \omega_{n}\right)\right)$ containing $\tilde{X}_{m+1}+$ $\omega_{m+1}\left(\right.$ recall $\left.I_{m+1}=\tilde{X}_{m+1}+[-\varepsilon, \varepsilon]\right)$.

${ }^{3}$ For our purposes, an interval is a bounded, connected subset of $\mathbb{R}$, with either open or closed endpoints. Since we care only about $\mathbb{P}$-typical trajectories, we need not specify what to do with endpoints. 
(b) On the event $\left\{\tau_{i}=m\right\}$, we have $I_{m+1}=\tilde{X}_{m+1}+[-\varepsilon, \varepsilon]$ and

$$
\tau_{i+1}=m+\tau\left[I_{m+1}\right]\left(\tilde{X}_{m+1}+\omega_{m+1}, \underline{\hat{\omega}}\right),
$$

where $\tau\left[I_{m+1}\right]$ is the stopping time as defined in Section 4.1 with $\underline{\hat{\omega}}=\left(0, \omega_{m+2}\right.$, $\left.\omega_{m+3}, \ldots\right)$.

\subsection{Filtration $\left(\mathscr{H}_{n}\right)$}

We now construct $\mathscr{H}_{n}=\sigma\left(\mathcal{A}_{n}\right)$, where the measurable partition $\mathcal{A}_{n}$ on $\Omega$ is defined below. Each $\mathcal{A}_{n}$ will consist of $\mathcal{F}_{n}$-measurable atoms, and so will be treated here as a partition on the first $n+1$ coordinates $\left(\omega_{0}, \ldots, \omega_{n}\right) \in[-\varepsilon, \varepsilon]^{n+1}$.

To start, we set $\mathcal{A}_{0}=\{[-\varepsilon, \varepsilon]\}$ to be the trivial partition, and hereafter assume $n \geq 1$.

Continuing, for each $i \geq 0$ and $0 \leq m<n$, the event $S_{i, m, n}$ (notation as in Lemma 5.2) can be treated as a subset of $[-\varepsilon, \varepsilon]^{n+1}$ since each $\tau_{i}$ is a stopping time with respect to $\mathscr{F}_{n}=\sigma\left(\omega_{0}, \ldots, \omega_{n}\right)$ (i.e., we have $\left\{\tau_{i}>n\right\} \in \mathscr{F}_{n}$ for all $\left.i, n\right)$. Define as well the events $S_{i, n}=\left\{\tau_{i}=n-1\right\}$, and observe that the collection

$$
\mathfrak{P}_{n}=\left\{S_{i, n}: i \geq 1\right\} \cup\left\{S_{i, m, n}: i \geq 1,0 \leq m<n\right\}
$$

is a partition of $[-\varepsilon, \varepsilon]^{n+1}$. We define $\mathcal{A}_{n} \geq \mathfrak{P}_{n}$ on each $\mathfrak{P}_{n}$-atom separately.

- For each set of the form $S_{i, m, n} \in \mathfrak{P}_{n}, i \geq 0,0 \leq m<n$, we define $\left.\mathcal{A}_{n}\right|_{S_{i, m, n}}$ to consist of atoms of the form

$$
\left\{\omega_{0}\right\} \times\left\{\omega_{1}\right\} \times \cdots \times\left\{\omega_{m}\right\} \times J \times\left\{\omega_{m+2}\right\} \times \cdots \times\left\{\omega_{n}\right\},
$$

as $J$ ranges over the atoms of $\mathcal{Q}_{n-m+1}\left(I_{m+1} ;\left(0, \omega_{m+2}, \ldots, \omega_{n}\right)\right)$. Here we identify $[-\varepsilon, \varepsilon]$ with $I_{m+1}=\widetilde{X}_{m+1}+[-\varepsilon, \varepsilon]$ in the obvious way.

- On each set $S_{i, n} \in \mathfrak{P}_{n}, i \geq 1$, we define $\left.\mathcal{A}\right|_{S_{i, n}}$ to consist of atoms of the form

$$
\left\{\omega_{0}\right\} \times\left\{\omega_{1}\right\} \times \cdots \times\left\{\omega_{n-1}\right\} \times[-\varepsilon, \varepsilon] .
$$

With $\mathscr{A}_{n}$ completely described, the construction of $\mathscr{H}_{n}:=\sigma\left(\mathscr{A}_{n}\right)$ is complete. It is not hard to check that $\mathscr{H}_{n}$ is a filtration, i.e., $\mathscr{H}_{n} \supset \mathscr{H}_{n-1}$ : to do this, one verifies that the partition sequence $\mathcal{A}_{n}$ is increasing by inspecting each $\mathfrak{B}_{n}$-atom separately.

The following is a straightforward consequence of Lemma 5.2.

Lemma 5.3. For each $n \geq 1$, the random interval $I_{n}$ is $\mathscr{H}_{n}$-measurable. Moreover, the measure $v_{n}(\cdot)=\mathbb{P}\left(\tilde{X}_{n} \in \cdot \mid \mathscr{H}_{n}\right)$ satisfies $\operatorname{supp}\left(v_{n}\right)=I_{n}$.

\subsection{The conditional measures $v_{n}$}

Let us first describe more transparently what the conditional measures $v_{n}(\cdot)=\mathbb{P}\left(\tilde{X}_{n} \in\right.$ $\left.\cdot \mid \mathscr{H}_{n}\right)$ actually are. To start, for $\underline{\omega} \in S_{i, n}, i \geq 0, n \geq 1$, we have that $v_{n}=\delta_{\tilde{X}_{n}} * v^{\varepsilon}$ is the uniform distribution on $I_{n}=\tilde{X}_{n}+[-\varepsilon, \varepsilon]$. The following characterizes $v_{n}$ on the event $S_{i, m, n}, i \geq 0,0 \leq m<n$. 
Lemma 5.4. Let $i \geq 0,0 \leq m<n$ and condition on the event $S_{i, m, n}=\left\{\tau_{i}=m\right.$, $\left.\tau_{i+1} \geq n\right\}$. Define $\widehat{F}_{m, n}:[-\varepsilon, \varepsilon] \rightarrow \mathbb{R}$ to be the map sending $\omega \mapsto \tilde{X}_{n}=\tilde{f}_{\omega_{n-1}} \circ \cdots \circ$ $\tilde{f}_{\omega_{m+2}} \circ \tilde{f}_{\omega}\left(\tilde{X}_{m+1}\right)$.

Let $J \in \mathcal{Q}_{n-m-1}\left(\tilde{X}_{m+1} ;\left(0, \omega_{m+2}, \ldots, \omega_{n}\right)\right)$ (regarded as a partition of $\left.[-\varepsilon, \varepsilon]\right)$ be the atom containing $\omega_{m+1}$. Then $\widehat{F}_{m, n}: J \rightarrow I_{n}$ is a diffeomorphism, and

$$
v_{n}=\frac{1}{v^{\varepsilon}(J)}\left(\widehat{F}_{m, n}\right)_{*}\left(\left.v^{\varepsilon}\right|_{J}\right)
$$

The proof is a case-by-case verification of the above formula and is left to the reader.

Recall that $J \subset[-\varepsilon, \varepsilon]$ appearing in (13) has the property that points in $\tilde{X}_{m+1}+J$ have the same itinerary under $\tilde{f}_{\theta^{m+2} \omega}^{n-m-1} \circ \tilde{f}$. In that notation, we have that the density $\rho_{n}=\frac{d v_{n}}{d \text { Leb }}$ at a point $x \in I_{n}$ is, up to a constant scalar, given by

$$
\left(\widehat{F}_{m, n}\right)^{\prime}(\omega)=\left(f_{\theta^{m+2} \underline{\omega}}^{n-m-1} \circ f\right)^{\prime}\left(\widetilde{X}_{m+1}+\omega\right),
$$

where $\omega \in[-\varepsilon, \varepsilon]$ is such that $x=\widehat{F}_{m, n}(\omega)$. In view of Proposition 4.5 and Lemma 5.2, then, we obtain a distortion estimate for the density $\rho_{n}=\frac{d v_{n}}{d \text { Leb }}$.

Corollary 5.5. Let $n \geq 1$ be such that $I_{n}$ is free. Then, for all $x, x^{\prime} \in I_{n}$, we have the estimate

$$
\frac{\rho_{n}(x)}{\rho_{n}\left(x^{\prime}\right)} \leq \exp \left(K_{2} L^{-1 / 2}+4\left\|\psi^{\prime \prime}\right\|_{C^{0}} L^{2 \beta}\left|x-x^{\prime}\right|\right)
$$

\section{Lyapunov exponents}

Finally, we come to the estimation of Lyapunov exponents in Theorem B. Throughout, we assume the setup of Section 2.1.1 and that $\varepsilon \geq L^{-(2 k+1)(1-\beta)+\alpha}$ for some $\alpha \geq 0$. By Theorem A, it follows that there is a unique ergodic stationary measure $\mu$ supported on $S^{1}$.

By (a version of) the Birkhoff ergodic theorem (see [28, Corollary 2.2 on p. 24]), we have that

$$
\lambda=\lim _{n \rightarrow \infty} \frac{1}{n} \log \left|\left(f_{\underline{\omega}}^{n}\right)^{\prime}(x)\right|
$$

exists and is constant over $\mathbb{P}$-a.e. $\underline{\omega} \in \Omega$ and $\mu$-a.e. $x \in S^{1}$. Since, however, $\mu$ is absolutely continuous and supported on all of $S^{1}$, we can promote this limit to every $x \in S^{1}$ and $\mathbb{P}$ a.e. $\underline{\omega} \in \Omega$; details are left to the reader.

It remains to estimate $\lambda$ from below, for which we use the following.

Lemma 6.1. In the above setting, we have

$$
\lambda \geq \inf _{x \in S^{1}} \liminf _{n \rightarrow \infty} \frac{1}{n} \mathbb{E}\left(\log \left|\left(f_{\underline{\omega}}^{n}\right)^{\prime}(x)\right|\right)
$$

for all $x \in S^{1}$. 
Proof. The limit

$$
\lambda=\lim _{n \rightarrow \infty} \frac{1}{n} \int_{S^{1}} \mathbb{E}\left(\log \left|\left(f_{\underline{\omega}}^{n}\right)^{\prime}(x)\right|\right) d \mu(x)
$$

follows from the $L^{1}$-mean ergodic theorem applied to the skew product $\tau: S^{1} \times \Omega \rightarrow$ $S^{1} \times \Omega$ defined by setting $\tau(x, \underline{\omega})=\left(f_{\omega_{0}} x, \theta \underline{\omega}\right)$, on noting that $\mu$ is a stationary ergodic measure iff $\mu \otimes \mathbb{P}$ is an ergodic invariant measure for $\tau$ ([28, Theorem 2.1 on p. 20]).

As is not hard to check, for all $x \in S^{1}$ we have $-d(L, \varepsilon) \leq \mathbb{E}\left(\log \left|f_{\omega}^{\prime}(x)\right|\right) \leq \log L$ where $d(L, \varepsilon)>0$ is a constant depending only on $\varepsilon, L$. These bounds pass to the averages $g_{n}:=\frac{1}{n} \mathbb{E}\left(\log \left|\left(f_{\underline{\omega}}^{n}\right)^{\prime}(x)\right|\right)$. Applying Fatou's lemma to the nonnegative sequence $g_{n}+$ $d(L, \varepsilon)$, we conclude

$$
\inf _{x \in S^{1}} \liminf _{n \rightarrow \infty} \frac{1}{n} \mathbb{E}\left(\log \left|\left(f_{\underline{\omega}}^{n}\right)^{\prime}(x)\right|\right) \leq \int_{S^{1}} \liminf _{n} g_{n} d \mu \leq \lim _{n \rightarrow \infty} \int_{S^{1}} g_{n} d \mu=\lambda .
$$

The remaining work is to estimate $\liminf _{n} \frac{1}{n} \mathbb{E}\left(\log \left|\left(f_{\underline{\omega}}^{n}\right)^{\prime}(x)\right|\right)$ for arbitrary $x \in S^{1}$.

Proposition 6.2. For all $x \in S^{1}$, we have

$$
\liminf _{n \rightarrow \infty} \frac{1}{n} \mathbb{E}\left(\log \left|\left(f_{\underline{\omega}}^{n}\right)^{\prime}(x)\right|\right) \geq \lambda_{0} \log L,
$$

where $\lambda_{0}=\min \left\{\frac{\alpha}{k+1}, \frac{1}{10}\right\}$.

The proof of Proposition 6.2 occupies the remainder of Section 6.

Reductions. We make here some slight modifications to the upper and lower bounds on $\varepsilon$ and the parameter $\beta$. To start, on shrinking the parameter $\beta$, we assume

$$
\varepsilon \geq L^{-\frac{1-\beta}{1+\beta} k-(1-\beta)(k+1)+\alpha} .
$$

Second, we can assume without loss that $\varepsilon<L^{-\min \left\{k-1, \frac{1}{2}\right\}}$ as in the hypothesis (3) for Lemma 2.1. If not, then we can reduce to this case by a similar line of reasoning as to the reductions in Section 3.2 in the proof of Theorem A, to which we refer for details.

Finally, a minor technical point: we will assume that $k, \beta$ satisfy the relation

$$
\left(\frac{3}{10}-\frac{5}{2} \beta-\beta^{2}\right) k \geq 2 \beta(1+\beta) .
$$

For $k \geq 6$, (15) is automatic for all $\beta \in\left(0, \frac{1}{10}\right)$, while (15) holds for all $k \in \mathbb{N}$ when $\beta \in\left(0, \frac{1}{100}\right)$. This entails no loss of generality.

With $\beta$ fixed once and for all, we let $L$ be sufficiently large, in terms of $\beta$, and take on the assumptions of Section 2.1.1. The parameter $\varepsilon$ is as above, and for our choice of $k \in \mathbb{N}$ we assume (15) holds. Finally, the constructions of Section 5 (namely, the filtration $\left.\mathscr{H}_{n}\right)$ are applied to the arbitrary initial condition $x=X_{0} \in S^{1}$. 


\subsection{Decomposing the sum}

Fix $n \geq 1$. Define $T_{i}=\log \left|\tilde{f}_{\omega_{i}}^{\prime}\left(X_{i}\right)\right|, X_{i}:=f_{\underline{\omega}}^{i}(x)$. With $\tau_{0} \equiv 0<\tau_{1}<\tau_{2}<\cdots$ as in Section 5 , define the random index $J \in \mathbb{Z}_{\geq 0}$ to satisfy

$$
\tau_{J}<n \leq \tau_{J+1}
$$

note that $\tau_{1} \geq n$ implies $J=0$ since $\tau_{0}:=0$.

We decompose

$$
(*):=\log \left|\left(f_{\underline{\omega}}^{n}\right)^{\prime}(x)\right|=\sum_{i=0}^{n-1} T_{i}=\sum_{i=0}^{\min \left\{\tau_{1}, n\right\}-1} T_{i}+\sum_{j=1}^{\infty} \chi_{J \geq j}\left(T_{\tau_{j}}+\sum_{i=\tau_{j}+1}^{\min \left\{\tau_{j+1}, n\right\}-1} T_{i}\right)
$$

and will bound $\mathbb{E}(*)$ from below; here, for an event $A$ we write $\chi_{A}$ for the indicator function of $A$. The main obstacles are the terms $T_{\tau_{j}}, 1 \leq j \leq J$, which we bound from below using conditional expectations with respect to the filtration $\left(\mathscr{H}_{n}\right)_{n}$.

Proposition 6.3. Let $j \geq 2$ and condition on the event $\tau_{j}=m$. Then

$$
\mathbb{E}\left(T_{m} \mid \mathscr{H}_{m}\right) \geq-\gamma \log L
$$

where $\gamma:=\max \left\{(1+\beta)\left(\left(\frac{1}{2}+\beta\right) k+2 \beta\right), k(1-\beta)-\alpha\right\}$.

Proposition 6.3 is proved in Section 6.2.

We apply Proposition 6.3 by replacing the terms $\chi_{J \geq j} T_{\tau_{j}}, j \geq 2$ under $\mathbb{E}$ with the conditional expectations ${ }^{4}$

$$
(*)_{j}:=\mathbb{E}\left(\chi_{J \geq j} T_{\tau_{j}} \mid \mathscr{H}_{\tau_{j}}\right)=\sum_{m=1}^{n-1} \mathbb{E}\left(\chi_{\tau_{j}=m} T_{m} \mid \mathscr{H}_{m}\right)=\sum_{m=1}^{n-1} \chi_{\tau_{j}=m} \cdot \mathbb{E}\left(T_{m} \mid \mathscr{H}_{m}\right) .
$$

Here, we use that $\{J \geq j\}=\bigcup_{m=1}^{n-1}\left\{\tau_{j}=m\right\}$ for all $j \geq 1$. By Proposition 6.3 , for $j \geq 2$ we have

$$
(*)_{j} \geq-\gamma \log L \cdot \chi_{J \geq j} .
$$

For the $j=1$ term, we use the following crude estimate.

Lemma 6.4. We have

$$
(*)_{1}:=\mathbb{E}\left(\chi_{J \geq 1} T_{\tau_{1}} \mid \mathscr{H}_{\tau_{1}}\right) \geq-2(2 k+1) \log L=:-\gamma_{1} \log L
$$

We prove Lemma 6.4 in Section 6.2.

\footnotetext{
${ }^{4}$ For a filtration $\left(\boldsymbol{E}_{n}\right)$ and an adapted stopping time $\eta$, we write $\boldsymbol{\mathcal { E }}_{\eta}$ for the stopped $\sigma$-algebra consisting of the set of measurable sets $A$ for which $A \cap\{\eta \leq m\} \in \boldsymbol{E}_{m}$ for all $m$.
} 
Applying these estimates, we have

$$
\begin{aligned}
& \mathbb{E}(*) \geq \mathbb{E}\left[\sum_{i=0}^{\min \left\{\tau_{1}, n\right\}-1} T_{i}+(*)_{1}+\chi_{J \geq 1} \sum_{i=\tau_{1}+1}^{\min \left\{\tau_{2}, n\right\}-1} T_{i}\right. \\
& \left.+\sum_{j=2}^{\infty}\left((*)_{j}+\chi_{J \geq j} \sum_{i=\tau_{j}+1}^{\min \left\{\tau_{j+1}, n\right\}-1} T_{i}\right)\right] \\
& \geq \mathbb{E}[\underbrace{\sum_{i=0}^{\min \left\{\tau_{1}, n\right\}-1} T_{i}}_{\mathrm{I}}+\underbrace{\chi_{J \geq 1}\left(-\gamma_{1} \log L+\sum_{i=\tau_{1}+1}^{\min \left\{\tau_{2}, n\right\}-1} T_{i}\right)}_{\mathrm{II}} \\
& +\underbrace{\sum_{j=2}^{\infty} \chi_{J \geq j} \cdot\left(-\gamma \log L+\sum_{i=\tau_{j}+1}^{\min \left\{\tau_{j+1}, n\right\}-1} T_{i}\right)}_{\mathrm{III}} \\
& =: \mathbb{E}[\mathrm{I}+\mathrm{II}+\mathrm{III}] \text {. }
\end{aligned}
$$

To complete the estimate, we decompose according to the events $\{J=K\}, K=0,1,2, \ldots$

(A) Estimate of $\mathbb{E}\left(\chi_{J=0}(\mathbf{I}+\mathbf{I I}+\mathbf{I I I})\right)$. We have II $=$ III $=0$ and

$$
\mathbb{E}\left[\chi_{J=0} \cdot \mathrm{I}\right]=\mathbb{E}\left[\chi_{J=0} \sum_{i=0}^{n-1} T_{i}\right]
$$

Conditioned on $J=0$, we have $\tau_{1} \geq n$ and so Lemma 4.4 may be applied (see also Lemma 5.2). We obtain a lower bound using the worst possible case that $p_{\omega_{n-1}}\left(I_{n-1}\right)=k-1$, i.e., $I_{n-1}$ initiates a bound period of length $k-1$ at time $n-1$ (corresponding to $t_{j}=n-1$, $p_{j}=k-1$ in the notation of Lemma $\left.4.4(\mathrm{~b})\right)$. So,

$$
\sum_{i=0}^{n-1} T_{i}=\log \left|\left(f_{\underline{\omega}}^{n}\right)^{\prime}\left(x_{0}\right)\right| \geq L^{(n-1)\left(\frac{1}{2}-\beta\right)-\frac{k-1}{2}-\beta} .
$$

We conclude

$$
\mathbb{E}\left[\chi_{J=0} \cdot \mathrm{I}\right] \geq\left((n-1)\left(\frac{1}{2}-\beta\right) \log L-\left(\frac{k-1}{2}+\beta\right) \log L\right) \cdot \mathbb{P}(J=0) .
$$

(B) Estimate of $\mathbb{E}\left(\chi_{J=1}(\mathbf{I}+\mathbf{I I}+\mathbf{I I I})\right)$. Here we have III $=0$ and

$$
\mathbb{E}\left[\chi_{J=1} \cdot(\mathrm{I}+\mathrm{II})\right]=\mathbb{E}\left[\chi_{J=1}\left(\sum_{i=0}^{\tau_{1}-1} T_{i}-\gamma_{1} \log L+\sum_{i=\tau_{1}+1}^{n-1} T_{i}\right)\right]
$$

By Lemma 4.4(a) we have $\sum_{i=0}^{\tau_{1}-1} T_{i} \geq \tau_{1} \cdot\left(\frac{1}{2}-\beta\right) \log L$. The second summation $\sum_{i=\tau_{1}+1}^{n-1} T_{i}$ is estimated as in paragraph (A): we have

$\mathbb{E}\left[\chi_{J=1} \sum_{i=\tau_{1}+1}^{n-1} T_{i}\right] \geq\left(\left(n-2-\tau_{1}\right)\left(\frac{1}{2}-\beta\right) \log L-\left(\frac{k-1}{2}+\beta\right) \log L\right) \cdot \mathbb{P}(J=1)$, 
and so collecting, we get

$\mathbb{E}\left[\chi_{J=1} \cdot(\mathrm{I}+\mathrm{II})\right] \geq\left((n-2)\left(\frac{1}{2}-\beta\right) \log L-\gamma_{1} \log L+\left(1-\frac{k}{2}-\beta\right) \log L\right) \cdot \mathbb{P}(J=1)$.

(C) Estimate of $\mathbb{E}\left(\chi_{J=K}(\mathbf{I}+\mathbf{I I}+\right.$ III $\left.)\right)$ for $K>1$. We bound $\mathbb{E}\left[\chi_{J=K} \cdot(\mathrm{I}+\mathrm{II})\right]$ as in paragraph (A), obtaining

$$
\mathbb{E}\left[\chi_{J=K} \cdot(\mathrm{I}+\mathrm{II})\right] \geq \mathbb{E}\left[\chi_{J=K}\left(\left(\tau_{2}-1\right)\left(\frac{1}{2}-\beta\right) \log L-\gamma_{1} \log L\right)\right] .
$$

Conditioned on $\{J=K\}$ for $K>1$, the III term has the form

$$
\mathrm{III}=\sum_{j=2}^{K-1} \underbrace{\left(-\gamma \log L+\sum_{i=\tau_{j}+1}^{\tau_{j+1}-1} T_{i}\right)}_{\mathrm{IV}_{j}}+\underbrace{\left(-\gamma \log L+\sum_{i=\tau_{K}+1}^{n-1} T_{i}\right)}_{\mathrm{IV}_{K}} .
$$

For each summand $\mathrm{IV}_{j}, j \geq 2$, observe that $\tilde{X}_{i} \in \mathcal{E}$ for each $i=\tau_{j}+1, \ldots, \tau_{j}+k$, hence $\sum_{i=\tau_{j}+1}^{\tau_{j}+k} T_{i} \geq k(1-\beta) \log L$. If $\tau_{j}+k+1 \leq \tau_{j+1}-1$, then the summands $\tau_{j}+k+1 \leq$ $i \leq \tau_{j+1}-1$ are estimated as in Lemma 4.4(a). In total,

$$
\begin{aligned}
\mathbb{E}\left[\chi_{J}\right. & \left.=K \cdot \mathrm{IV}_{j}\right] \\
& \geq \mathbb{E}\left[\chi_{J=K}\left((k(1-\beta)-\gamma) \cdot \log L+\left(\tau_{j+1}-1-\tau_{j}-k\right) \cdot\left(\frac{1}{2}-\beta\right) \log L\right)\right] .
\end{aligned}
$$

Observe that

$$
k(1-\beta)-\gamma=\min \left\{\alpha,\left(\frac{1}{2}-\frac{5}{2} \beta-\beta^{2}\right) k-2 \beta(1+\beta)\right\} \geq \min \left\{\alpha(k+1), \frac{1}{5} k\right\}
$$

holds from (15). Dividing the latter by $k+1$ yields an estimate for the average growth rate $\lambda_{0}$ as follows:

$$
\frac{k(1-\beta)-\gamma}{k+1} \geq \min \left\{\alpha, \frac{1}{10}\right\}=: \lambda_{0}=\lambda_{0}(\alpha, k),
$$

hence

$$
\mathbb{E}\left[\chi_{J=K} \cdot \mathrm{IV}_{j}\right] \geq \mathbb{E}\left[\chi_{J=K}\left(\tau_{j+1}-\tau_{j}\right) \cdot \lambda_{0} \log L\right] .
$$

This telescopes, and so

$$
\mathbb{E}\left[\chi_{J=K} \sum_{j=2}^{K-1} \mathrm{IV}_{j}\right] \geq \mathbb{E}\left[\chi_{J=K}\left(\tau_{K}-\tau_{2}\right) \cdot \lambda_{0} \log L\right] .
$$

Using Lemma 4.4 (b), we bound $\mathrm{IV}_{K}$ from below by

$$
\begin{aligned}
\mathrm{IV}_{K} & =-\gamma \log L+\sum_{j=\tau_{K}+1}^{n-1} T_{i} \\
& \geq-\gamma \log L+\left(n-\tau_{K}-2\right)\left(\frac{1}{2}-\beta\right) \log L-\left(\frac{k-1}{2}+\beta\right) \log L,
\end{aligned}
$$


hence

$$
\mathbb{E}\left[\chi_{J=K} \cdot \mathrm{III}\right] \geq \mathbb{E}\left[\chi_{J=K}\left(\left(n-2-\tau_{2}\right) \cdot \lambda_{0} \log L-\gamma \log L-\left(\frac{k-1}{2}+\beta\right) \log L\right)\right]
$$

and, in total,

$$
\begin{aligned}
\mathbb{E}\left[\chi_{J}=K\right. & (\mathrm{I}+\mathrm{II}+\mathrm{III})] \\
& \geq\left((n-3) \lambda_{0} \log L-\left(\gamma+\gamma_{1}\right) \log L-\left(\frac{k-1}{2}+\beta\right) \log L\right) \cdot \mathbb{P}(J=K) .
\end{aligned}
$$

Putting it together. The lower bounds obtained for $K>1$ as in paragraph (C) are the worst of the three cases examined already, hence

$$
\begin{aligned}
\mathbb{E}(*)=\mathbb{E}[\mathrm{I}+\mathrm{II}+\mathrm{III}] & =\sum_{K=0}^{\infty} \mathbb{E}\left[\chi_{J=K}(\mathrm{I}+\mathrm{II}+\mathrm{III})\right] \\
& \geq(n-3) \lambda_{0} \log L-\left(\gamma+\gamma_{1}\right) \log L-\left(\frac{k-1}{2}+\beta\right) \log L
\end{aligned}
$$

On dividing by $n$ and taking $n \rightarrow \infty$, we conclude that $\lim _{n \rightarrow \infty} \frac{1}{n} \mathbb{E}\left(\log \left|\left(f_{\underline{\omega}}^{n}\right)^{\prime}(x)\right|\right) \geq$ $\lambda_{0} \log L$, as desired.

\subsection{Proofs of Proposition 6.3 and Lemma 6.4}

Below, $C>0$ refers to a constant depending only on $\psi$, and may change in value from line to line.

We start with the following preliminary estimate.

Lemma 6.5. Let I $\subset \mathcal{B}$ be any connected interval. Then

$$
\int_{I} \log \left|f^{\prime}(z)\right| d z \geq|I| \cdot \log \left(L^{1-\beta}|I|\right) .
$$

This is a simple consequence of (1) and follows on taking $L$ sufficiently large, depending only on $\beta$ and $\psi$; details are left to the reader.

Proof of Proposition 6.3. Unconditionally, for any $m \geq 0$ the conditional expectation $\mathbb{E}\left(T_{m} \mid \mathscr{H}_{m}\right)$ is given by

$$
(* *)=\int_{I_{m}} \log \left|f_{\omega_{m}}^{\prime}(z)\right| d v_{m}(z)
$$

by Lemma 5.3 .

Conditioning on $\left\{\tau_{j}=m\right\}$, recall (Remark 4.2) that $\left|I_{m}\right| \leq C L^{-\frac{k}{2}-\beta}$ since $I_{m}$ is an atom of $\mathcal{P}_{\omega_{m}}\left(\tilde{f}_{\omega_{m-1}}\left(I_{m-1}\right)\right)$. Our distortion control on $\rho_{m}=\frac{d v_{m}}{d \text { Leb }}$ as in Corollary 5.5 along $I_{m}$ implies $\left|\log \frac{\rho_{m}(z)}{\rho_{m}\left(z^{\prime}\right)}\right| \leq K_{2} L^{-1 / 2}+2 K_{1}^{-1} L^{-\frac{k}{2}+\beta} \leq C L^{-1 / 2+\beta}$ for $z, z^{\prime} \in I_{m}$, hence

$$
(* *) \geq\left(1+C L^{-1 / 2+\beta}\right) \frac{1}{\left|I_{m}\right|}\left(\int_{I_{m}} \log \left|f_{\omega_{m+1}}^{\prime}(z)\right| d z\right) .
$$


From Lemma 6.5 applied to $I=I_{m}$, we conclude

$$
(* *) \geq\left(1+C L^{-1 / 2+\beta}\right) \log \left(L^{1-\beta}\left|I_{m}\right|\right) \geq(1+\beta) \log \left(L^{1-\beta}\left|I_{m}\right|\right) .
$$

We now bound $\left|I_{m}\right|$ from below.

Lemma 6.6. On the event $\left\{\tau_{j}=m\right\}, j \geq 2, m \geq 1$, we have the estimate

$$
\left|I_{m}\right| \geq \min \left\{L^{-1-\left(\frac{1}{2}+\beta\right) k-\beta}, L^{k(1-\beta)} \varepsilon\right\} .
$$

Assuming this and plugging in $\varepsilon \geq L^{-\frac{1-\beta}{1+\beta} k-(1-\beta)(k+1)+\alpha}$, we conclude

$$
\begin{aligned}
(* *) & \geq(1+\beta) \log \min \left\{L^{-\left(\frac{1}{2}+\beta\right) k-2 \beta}, L^{(k+1)(1-\beta)} \varepsilon\right\} \\
& \geq \min \left\{(1+\beta)\left(-2 \beta-\left(\frac{1}{2}+\beta\right)\right),(1+\beta)\left(\alpha-k \frac{1-\beta}{1+\beta}\right)\right\} \\
& \geq-\max \left\{(1+\beta)\left(\left(\frac{1}{2}+\beta\right) k+2 \beta\right), k(1-\beta)-\alpha\right\} \log L=:-\gamma \log L .
\end{aligned}
$$

To finish the proof of Proposition 6.3, it remains to prove Lemma 6.6.

Proof of Lemma 6.6. We distinguish two cases:

(a) $I_{i}=f_{\omega_{i-1}}\left(I_{i-1}\right)$ for each $\tau_{j-1}+2 \leq i \leq m=\tau_{j}$;

(b) $I_{i} \subsetneq f_{\omega_{i-1}}\left(I_{i-1}\right)$ for some $\tau_{j-1}+2 \leq i \leq m=\tau_{j}$.

In case (a), we easily have $\left|I_{\tau_{j-1}+k+1}\right| \geq L^{k(1-\beta)} \varepsilon$, and since no additional cuts are made, we estimate

$$
\begin{aligned}
\left|I_{m}\right| & =\left|\tilde{f}_{\omega_{m-1}} \circ \cdots \circ \tilde{f}_{\omega_{\tau_{j-1}+k+1}}\left(I_{\tau_{j-1}+k+1}\right)\right| \\
& \geq L^{\left(m-\left(\tau_{j-1}+k+1\right)\right)\left(\frac{1}{2}-\beta\right)}\left|I_{\tau_{j-1}+k+1}\right| \geq L^{k(1-\beta)} \varepsilon
\end{aligned}
$$

In case (b), set $i^{*}=\max \left\{i \leq \tau_{j}: I_{i} \subsetneq \tilde{f}_{\omega_{i-1}}\left(I_{i-1}\right)\right\}$ (note $i^{*}=m$ is possible), and note that if $i^{*}<m$ then

$$
I_{m}=\tilde{f}_{\omega_{m-1}} \circ \cdots \circ \tilde{f}_{\omega_{i}}\left(I_{i^{*}}\right) .
$$

To bound $\left|I_{i^{*}}\right|$ we split further to the cases (i) $p_{\omega_{i^{*}}}\left(I_{i^{*}}\right)=0$, (ii) $p_{\omega_{i^{*}}}\left(I_{i^{*}}\right) \in\{1, \ldots, k-1\}$ and (iii) $p_{\omega_{i}}\left(I_{i^{*}}\right)=k$. Note that in all cases, $\mathcal{P}_{\omega_{i *}}\left(\tilde{f}_{\omega_{i^{*}-1}}\left(I_{i^{*}-1}\right)\right)$ contains at least two elements, hence $I_{i}$ contains at least one atom of $\mathcal{P}_{\omega_{i} *}$ (Remark 4.2).

In case (b) (i), $I_{i^{*}} \subset \mathcal{I}_{\omega_{i *}} \cup \mathcal{E}_{\omega_{i *} *}$. Either $I_{i^{*}}$ contains an atom of $\mathscr{E}_{\omega_{i *} *}$, in which case $\left|I_{i^{*}}\right|$ is bounded from below by $\frac{1}{2} \min \left\{d\left(\hat{x}, \hat{x}^{\prime}\right): \hat{x}, \hat{x}^{\prime} \in C_{\psi}^{\prime}, \hat{x} \neq \hat{x}^{\prime}\right\}$, or $I_{i}$ * contains an atom of $\mathcal{P}_{\omega_{i} *} \mid I_{\omega_{i *}}$, hence $\left|I_{i^{*}}\right| \geq L^{-\frac{3}{2}-\beta}$ (the latter bound being the worse of the two). Since $I_{m}=I_{\tau_{j}}$ is free, we conclude $\left|I_{m}\right| \geq\left|I_{i^{*}}\right| \geq L^{-\frac{3}{2}-\beta}$ from Lemma 4.4 (a).

In case (b) (ii), we have automatically that $I_{i}$ is free and initiates a bound period of length $p^{*}=p_{\omega_{i *}}\left(I_{i^{*}}\right)$. Since $0<p^{*}<k-1$ by assumption, we cannot have $i^{*}=\tau_{j}=m$ (since then $p^{*}=k$ ) and so conclude $i^{*}<\tau_{j}$ in this case -indeed, we have $i^{*}+p^{*}+1 \leq$ $m=\tau_{j}$, since $I_{\tau_{j}}$ is free. From Remark 4.2 we have

$$
\left|I_{i}\right| \geq\left(p^{*}+1\right)^{-2} L^{-\frac{p^{*}+3}{2}-\beta} \geq L^{-\frac{p^{*}+3}{2}-\beta\left(p^{*}+1\right)},
$$


on taking $L$ large enough so $\beta>2 / \log L$. Moreover, since $I_{m}=I_{\tau_{j}}$ is free, we have

$$
\begin{aligned}
\left|I_{m}\right| \geq\left|I_{i^{*}+p^{*}+1}\right|=\left|\tilde{f}_{\theta^{i^{*}} \underline{\omega}}^{p^{*}+1}\left(I_{i^{*}}\right)\right| & \geq L^{\left(p^{*}+1\right)\left(\frac{1}{2}-\beta\right)}\left|I_{i^{*}}\right| \\
& \geq L^{\left(p^{*}+1\right)\left(\frac{1}{2}-\beta\right)} \cdot L^{-\frac{p^{*}+3}{2}-\beta\left(p^{*}+1\right)} \\
& =L^{-1-2 \beta\left(p^{*}+1\right)} .
\end{aligned}
$$

The worst possible case is $p^{*}=k-1$, and so we conclude $\left|I_{m}\right| \geq L^{-1-2 \beta k}$ in case (ii).

In case (b) (iii), we have necessarily that $i^{*}=m=\tau_{j}$. In the worst case, $I_{m}$ contains an atom of $\left.\mathcal{P}_{\omega_{m}}\right|_{\mathcal{B}_{\omega_{m}}^{k-1}}$, and so $\left|I_{m}\right| \geq k^{-2} L^{-\frac{k+2}{2}-\beta} \geq L^{-1-\left(\frac{1}{2}+\beta\right) k-\beta}$.

Proof of Lemma 6.4. Arguing in parallel to the proof of Proposition 6.3 (see (18)) we have, on the event $\left\{\tau_{1}=m\right\}$, the estimate

$$
\mathbb{E}\left(T_{m} \mid \mathscr{H}_{m}\right) \geq(1+\beta) \log \left(L^{1-\beta}\left|I_{m}\right|\right) .
$$

As before, we estimate $\left|I_{m}\right|$ from below.

Lemma 6.7. On the event $\left\{\tau_{1}=m\right\}$, we have the estimate $\left|I_{m}\right| \geq \min \left\{L^{-1-\left(\frac{1}{2}+\beta\right) k-\beta}, \varepsilon\right\}$.

Assuming this, we easily obtain

$$
\mathbb{E}\left(T_{m} \mid \mathscr{H}_{m}\right) \geq(1+\beta) \log \left(L^{1-\beta} \min \left\{L^{-1-\left(\frac{1}{2}+\beta\right) k-\beta}, \varepsilon\right\}\right) \geq-2(2 k+1) \log L,
$$

as claimed. It remains to prove Lemma 6.7.

Proof of Lemma 6.7. Condition on $\tau_{1}=m$. The proof is very much parallel to that of Lemma 6.6. Case (b) can be repeated verbatim, and yields the identical estimate $\left|I_{m}\right| \geq$ $L^{-1-\left(\frac{1}{2}+\beta\right) k-\beta}$.

The only difference is in case (a). Here, we observe that $I_{m}$ must be free, and so (Lemma 4.4 (a)) we have

$$
\left|I_{m}\right| \geq L^{m\left(\frac{1}{2}-\beta\right)} \cdot 2 \varepsilon \geq \varepsilon .
$$

This completes the proof of Lemma 6.7.

Funding. This material is based upon work supported by the National Science Foundation under Award No. DMS-1604805 (to A.B.) and Award No. DMS-2000167 (to Y.Y.).

\section{References}

[1] R. Aimino, H. Hu, M. Nicol, A. Török, and S. Vaienti, Polynomial loss of memory for maps of the interval with a neutral fixed point. Discrete Contin. Dyn. Syst. 35 (2015), no. 3, 793-806 Zbl 1351.37163 MR 3277171

[2] V. Baladi, M. Benedicks, and V. Maume-Deschamps, Decay of random correlation functions for unimodal maps. Rep. Math. Phys. 46 (2000), no. 1-2, 15-26 Zbl 1049.37003 MR 1803321 
[3] V. Baladi and L.-S. Young, On the spectra of randomly perturbed expanding maps. Comm. Math. Phys. 156 (1993), no. 2, 355-385 Zbl 0809.60101 MR 1233850

[4] M. Benedicks and L. Carleson, On iterations of $1-a x^{2}$ on $(-1,1)$. Ann. of Math. (2) 122 (1985), no. 1, 1-25 Zbl 0597.58016 MR 799250

[5] M. Benedicks and L. Carleson, The dynamics of the Hénon map. Ann. of Math. (2) 133 (1991), no. 1, 73-169 Zbl 0724.58042 MR 1087346

[6] M. Benedicks and L. Palmisano, Coexistence phenomena in the Hénon family. 2018, arXiv: 1811.00517

[7] M. Benedicks and M. Viana, Random perturbations and statistical properties of Hénon-like maps. Ann. Inst. H. Poincaré Anal. Non Linéaire 23 (2006), no. 5, 713-752 Zbl 1131.37033 MR 2259614

[8] M. Benedicks and L.-S. Young, Absolutely continuous invariant measures and random perturbations for certain one-dimensional maps. Ergodic Theory Dynam. Systems 12 (1992), no. 1, 13-37 Zbl 0769.58051 MR 1162396

[9] A. Blumenthal, J. De Simoi, and K. Zhang, Diffusion limit for a slow-fast standard map. Comm. Math. Phys. 374 (2020), no. 1, 187-210 Zbl 1452.37017 MR 4066589

[10] A. Blumenthal, J. Xue, and Y. Yang, Lyapunov exponents for random perturbations of coupled standard maps. 2020, arXiv:2004.10626

[11] A. Blumenthal, J. Xue, and L.-S. Young, Lyapunov exponents for random perturbations of some area-preserving maps including the standard map. Ann. of Math. (2) 185 (2017), no. 1, 285-310 Zbl 1365.37039 MR 3583355

[12] A. Blumenthal, J. Xue, and L.-S. Young, Lyapunov exponents and correlation decay for random perturbations of some prototypical 2D maps. Comm. Math. Phys. 359 (2018), no. 1, 347-373 Zbl 1392.37044 MR 3781453

[13] A. Blumenthal and L.-S. Young, Equivalence of physical and SRB measures in random dynamical systems. Nonlinearity 32 (2019), no. 4, 1494-1524 Zbl 1423.37023 MR 3925383

[14] J. Buzzi, No or infinitely many a.c.i.p. for piecewise expanding $C^{r}$ maps in higher dimensions. Comm. Math. Phys. 222 (2001), no. 3, 495-501 Zbl 1001.37003 MR 1888086

[15] B. V. Chirikov, A universal instability of many-dimensional oscillator systems. Phys. Rep. 52 (1979), no. 5, 264-379 MR 536429

[16] P. Collet and J.-P. Eckmann, Positive Liapunov exponents and absolute continuity for maps of the interval. Ergodic Theory Dynam. Systems 3 (1983), no. 1, 13-46 Zbl 0532.28014 MR 743027

[17] P. Duarte, Plenty of elliptic islands for the standard family of area preserving maps. Ann. Inst. H. Poincaré Anal. Non Linéaire 11 (1994), no. 4, 359-409 Zbl 0838.58030 MR 1287238

[18] P. Duarte, Elliptic isles in families of area-preserving maps. Ergodic Theory Dynam. Systems 28 (2008), no. 6, 1781-1813 Zbl 1179.37007 MR 2465600

[19] I. ElShaarawy and W. Gomaa, An efficient computational framework for studying dynamical systems. In 2013 15th International Symposium on Symbolic and Numeric Algorithms for Scientific Computing, pp. 138-145, IEEE, 2013

[20] Z. Galias, Systematic search for wide periodic windows and bounds for the set of regular parameters for the quadratic map. Chaos 27 (2017), no. 5, Art. ID 053106 Zbl 1387.37036 MR 3648847

[21] Z. Galias and W. Tucker, On the structure of existence regions for sinks of the Hénon map. Chaos 24 (2014), no. 1, Art. ID 013120 Zbl 1374.37098 MR 3402644

[22] Z. Galias and W. Tucker, Is the Hénon attractor chaotic? Chaos 25 (2015), no. 3, Art. ID 033102 Zbl 1374.37099 MR 3389819 
[23] A. Golmakani, C. E. Koudjinan, S. Luzzatto, and P. Pilarczyk, Rigorous numerics for critical orbits in the quadratic family. Chaos 30 (2020), no. 7, Art. ID 073143 Zbl 1453.37077 MR 4129310

[24] A. Gorodetski, On stochastic sea of the standard map. Comm. Math. Phys. 309 (2012), no. 1, 155-192 Zbl 1347.37049 MR 2864790

[25] M. Hénon, A two-dimensional mapping with a strange attractor. Comm. Math. Phys. 50 (1976), no. 1, 69-77 Zbl 0576.58018 MR 422932

[26] M. V. Jakobson, Absolutely continuous invariant measures for one-parameter families of onedimensional maps. Comm. Math. Phys. 81 (1981), no. 1, 39-88 Zbl 0497.58017 MR 630331

[27] A. Katok and Y. Kifer, Random perturbations of transformations of an interval. J. Analyse Math. 47 (1986), 193-237 Zbl 0616.60064 MR 874051

[28] Y. Kifer, Ergodic theory of random transformations. Progress in Probability and Statistics 10, Birkhäuser Boston, Boston, MA, 1986 Zbl 0604.28014 MR 884892

[29] Y. Kifer, Random perturbations of dynamical systems. Progress in Probability and Statistics 16, Birkhäuser Boston, Boston, MA, 1988 Zbl 0659.58003 MR 1015933

[30] E. N. Lorenz, The nature and theory of the general circulation of the atmosphere, vol. 218, World Meteorological Organization, Geneva, 1967

[31] F. Ledrappier, M. Shub, C. Simó, and A. Wilkinson, Random versus deterministic exponents in a rich family of diffeomorphisms. J. Statist. Phys. 113 (2003), no. 1-2, 85-149 Zbl 1031.37028 MR 2012975

[32] F. Ledrappier and L.-S. Young, Entropy formula for random transformations. Probab. Theory Related Fields 80 (1988), no. 2, 217-240 Zbl 0638.60054 MR 968818

[33] Z. Lian and M. Stenlund, Positive Lyapunov exponent by a random perturbation. Dyn. Syst. 27 (2012), no. 2, 239-252 Zbl 1293.37023 MR 2926701

[34] K. Lu, Q. Wang, and L.-S. Young, Strange attractors for periodically forced parabolic equations. Mem. Amer. Math. Soc. 224 (2013), no. 1054 Zbl 1341.37050 MR 3098967

[35] S. Luzzatto and P. Pilarczyk, Finite resolution dynamics. Found. Comput. Math. 11 (2011), no. 2, 211-239 Zbl 1211.65164 MR 2776399

[36] S. Luzzatto and H. Takahasi, Computable conditions for the occurrence of non-uniform hyperbolicity in families of one-dimensional maps. Nonlinearity 19 (2006), no. 7, 1657-1695 Zbl 1113.37021 MR 2229798

[37] M. Lyubich, Almost every real quadratic map is either regular or stochastic. Ann. of Math. (2) 156 (2002), no. 1, 1-78 Zbl 1160.37356 MR 1935840

[38] M. Misiurewicz, Absolutely continuous measures for certain maps of an interval. Inst. Hautes Études Sci. Publ. Math. (1981), no. 53, 17-51 Zbl 0477.58020 MR 623533

[39] L. Mora and M. Viana, Abundance of strange attractors. Acta Math. 171 (1993), no. 1, 1-71 Zbl 0815.58016 MR 1237897

[40] S. E. Newhouse, Diffeomorphisms with infinitely many sinks. Topology 13 (1974), 9-18 Zbl 0275.58016 MR 339291

[41] S. E. Newhouse, The abundance of wild hyperbolic sets and nonsmooth stable sets for diffeomorphisms. Inst. Hautes Études Sci. Publ. Math. (1979), no. 50, 101-151 Zbl 0445.58022 MR 556584

[42] W. Ott and M. Stenlund, From limit cycles to strange attractors. Comm. Math. Phys. 296 (2010), no. 1, 215-249 Zbl 1202.37046 MR 2606633

[43] W. Ott, M. Stenlund, and L.-S. Young, Memory loss for time-dependent dynamical systems. Math. Res. Lett. 16 (2009), no. 3, 463-475 Zbl 1177.37055 MR 2511626 
[44] D. Ruelle, A review of linear response theory for general differentiable dynamical systems. Nonlinearity 22 (2009), no. 4, 855-870 Zbl 1158.37305 MR 2486360

[45] M. Stenlund and H. Sulku, A coupling approach to random circle maps expanding on the average. Stoch. Dyn. 14 (2014), no. 4, Art. ID 1450008 Zbl 1327.37012 MR 3261318

[46] W. Tucker and D. Wilczak, A rigorous lower bound for the stability regions of the quadratic map. Phys. D 238 (2009), no. 18, 1923-1936 Zbl 1197.37060 MR 2598514

[47] Q. Wang and W. Ott, Dissipative homoclinic loops of two-dimensional maps and strange attractors with one direction of instability. Comm. Pure Appl. Math. 64 (2011), no. 11, 14391496 Zbl 1229.37029 MR 2832166

[48] Q. Wang and L.-S. Young, Nonuniformly expanding 1D maps. Comm. Math. Phys. 264 (2006), no. 1, 255-282 Zbl 1107.37028 MR 2212223

[49] Q. Wang and L.-S. Young, Toward a theory of rank one attractors. Ann. of Math. (2) 167 (2008), no. 2, 349-480 Zbl 1181.37049 MR 2415378

[50] L.-S. Young, Stochastic stability of hyperbolic attractors. Ergodic Theory Dynam. Systems 6 (1986), no. 2, 311-319 Zbl 0633.58023 MR 857204

Received 31 August 2018; revised 24 April 2021; accepted 21 June 2021.

\section{Alex Blumenthal}

School of Mathematics, Georgia Institute of Technology, Atlanta, GA 30332, USA;

ablumenthal6@gatech.edu

\section{Yun Yang}

Department of Mathematics, Virginia Tech, Blacksburg, VA 24061, USA; yunyang@vt.edu 